Article

\title{
Visible Light as an Antimicrobial Strategy for Inactivation of Pseudomonas fluorescens and Staphylococcus epidermidis Biofilms
}

\author{
Valeria Angarano ${ }^{1}$, Cindy Smet $^{1}{ }^{1}$, Simen Akkermans ${ }^{1}{ }^{1}$, Charlotte Watt ${ }^{1}$, Andre Chieffi ${ }^{2}$ \\ and Jan F.M. Van Impe ${ }^{1, *(D)}$ \\ 1 BioTeC + , Chemical and Biochemical Process Technology and Control, Department of Chemical Engineering, \\ KU Leuven, 9000 Gent, Belgium; valeria.angarano@kuleuven.be (V.A.); cindy.smet@kuleuven.be (C.S.); \\ simen.akkermans@kuleuven.be (S.A.); charlotteg0306@gmail.com (C.W.) \\ 2 Procter \& Gamble, Newcastle Innovation Center, Newcastle NE12 9TS, UK; chieffi.a@pg.com \\ * Correspondence: jan.vanimpe@kuleuven.be; Tel.: +32-9-331-66-19 or +32-477-256-172
}

Received: 12 March 2020; Accepted: 7 April 2020; Published: 10 April 2020

check for updates

\begin{abstract}
The increase of antimicrobial resistance is challenging the scientific community to find solutions to eradicate bacteria, specifically biofilms. Light-Emitting Diodes (LED) represent an alternative way to tackle this problem in the presence of endogenous or exogenous photosensitizers. This work adds to a growing body of research on photodynamic inactivation using visible light against biofilms. Violet $(400 \mathrm{~nm})$, blue $(420 \mathrm{~nm})$, green $(570 \mathrm{~nm})$, yellow $(584 \mathrm{~nm})$ and red $(698 \mathrm{~nm})$ LEDs were used against Pseudomonas fluorescens and Staphylococcus epidermidis. Biofilms, grown on a polystyrene surface, were irradiated for $4 \mathrm{~h}$. Different irradiance levels were investigated $(2.5 \%, 25 \%, 50 \%$ and $100 \%$ of the maximum irradiance). Surviving cells were quantified and the inactivation kinetic parameters were estimated. Violet light could successfully inactivate P. fluorescens and S. epidermidis (up to 6.80 and $3.69 \log _{10}$ reduction, respectively), while blue light was effective only against $P$. fluorescens $(100 \%$ of maximum irradiance). Green, yellow and red irradiation neither increased nor reduced the biofilm cell density. This is the first research to test five different wavelengths (each with three intensities) in the visible spectrum against Gram-positive and Gram-negative biofilms. It provides a detailed study of the potential of visible light against biofilms of a different Gram-nature.
\end{abstract}

Keywords: Biofilm; photodynamic inactivation; visible light; light-emitting diode; polystyrene surface; inactivation kinetics; disinfection; antimicrobial inactivation; Pseudomonas fluorescens; Staphylococcus epidermidis

\section{Introduction}

Biofilms are bacterial communities, mono- or multi-species, embedded in a self-produced matrix mainly composed of proteins, polysaccharides, extracellular DNA and lipids, which constitute the extracellular polymeric substance (EPS) [1-6]. The EPS allows bacteria to adhere to many surfaces and it protects the community from hazardous compounds. This crucial layer of protection decreases the bacterial susceptibility to disinfectants and antibiotics $[7,8]$ and promotes cell communication via quorum sensing [9]. Biofilms living on surfaces have evolved to be more resistant to antibiotics, compared to their planktonic form $[10,11]$. They are identified as the major source of microbial contamination within the food industries, households and clinical environment [2]. Moreover, the development of antibiotic-resistant bacteria is raising a public health concern, and, at the same time, this underlines the urge for the scientific community to develop alternative antibacterial methods and strategies to fight infections [12]. Biofilms of different strains have been found in factories [13], 
meat, dairy, poultry industries [14], households [15], food processing industries [13] and hospitals [16]. They can resist harsh and hostile conditions, such as heat, low temperature, desiccation and shear forces, used as eradication methodologies [14,17]. Moreover, they cause issues ranging from process inefficiencies, such as corrosions or pipeline blockage [18], to infections due to their resistance to antibiotics [11].

Alternative, innovative and unconventional approaches are proposed to be used against microbial biofilms. These approaches can be based on the use of, e.g., plasma-based technologies [19], coatings embedding nanoparticles [20], ultrasound treatments [21], enzymatic disruption [22] or light-based technologies [23]. Within the light-technology framework, Light-Emitting Diodes (LEDs) are desirable for industries in terms of costs, safety, convenience and maintenance compared to other light sources, which have been used in different inactivation studies, e.g., lamps [24-26], lasers [27] or pulsed lights [28]. Moreover, they are characterized by narrow emission spectra allowing the selection of a specific range of wavelengths for irradiation. The use of visible light might represent an alternative way to effectively tackle biofilm problems, where antibiotics fail due to antimicrobial resistance. The photoeradication process is based on the absorption of photons by endogenous photosensitizers (PSs), when the microorganisms (fungi and bacteria) produce them, or exogenous PSs, when they are externally added before the irradiation process. After light excitation, the triplet state of these molecules results in the formation of reactive oxygen species [29-31]. The reactive oxygen species then cause damage to lipids, proteins, DNA, enzymes, RNA and other macromolecules and are therefore harmful to the microorganisms [32]. Some authors tried to quantify the endogenous PSs inside the bacteria [33-36], but the exact correlation between the light treatment efficiency and the number and type (structure, absorption and emission spectrum) of PSs within bacteria is still under investigation [37-42]. Based on some studies, the endogenous PSs absorbing light are ubiquinones, porphyrin-containing cytochromes or flavin-containing enzymes (having absorption spectra within 230-500 nm, $400-420 \mathrm{~nm}$ and $380-450 \mathrm{~nm}$, respectively). More specifically, the bacterial porphyrines extracted and found in the literature are protoporphyin IX, zinc protoporphyrin IX, coproporphyrin III and uroporphyrin III, which can coexist within the same strain [37,38,41]. Different types imply distinct chemical structures, spectra and quantum yield of reactive oxygen species production.

The sole use of light as an antimicrobial technology is particularly advantageous because it exploits the endogenous PSs that are naturally present inside the cells. It omits the use of additional chemicals and antibiotics, categorizing the technology as environmentally friendly [37]. The technology has the potential to stop the dispersion of antibiotics within the soil, caused by animal manure, for instance. It could reduce the quantities absorbed by humans and animals due to their presence within the food chain [12]. Moreover, the disuse of chemicals and antibiotics encourages to reduce both the use of resources and the production of chemical waste, having an economic impact. The visible spectrum is stated as harmless to humans, unlike UVB $(280-315 \mathrm{~nm})$ which directly damages DNA of both microbial and human cells [23], and it makes the technology suitable for household, industrial and hospital applications. Finally, some studies demonstrated that the light treatment does not cause the development of antimicrobial resistance thanks to the multi-target mechanism causing cell death $[25,37,43,44]$. The number of photodynamic inactivation studies has greatly increased in recent years. These studies have mainly examined planktonic cells [38-41,43,45-52], while few studies have taken bacterial biofilms into consideration [16,30,32,50-55]. Since biofilms pose a major global healthcare problem, the impact of photodynamic inactivation on them is both crucial and innovative. Previous research has found a bactericidal/inhibitory effect of light within the visible range on different species (Pseudomonas, Staphylococcus, Escherichia, Listeria, etc.) [27,30,32,46,48-51,56]. However, these studies are mainly focused on blue and violet light, even if there is previous evidence in the literature that green, yellow and red light had an impact on several bacterial species and fungi either promoting growth $[49,57,58]$ or decreasing the bacterial population $[24,30,46,48,49]$.

Since biofilms can directly be treated with a light source, regardless of the shape and the material composing the surface, the light treatment becomes simply applicable and advisable as new 
technology [51,59]. Indeed, this study adds to a growing body of research on photodynamic inactivation using visible light against biofilms. Thus, the impact of violet $(400 \mathrm{~nm})$, blue $(420 \mathrm{~nm})$, green $(570 \mathrm{~nm})$, yellow $(584 \mathrm{~nm})$ and red $(698 \mathrm{~nm})$ light with different irradiance levels $(25 \%, 75 \%$ and $100 \%$ of maximum irradiance $\left(\mathrm{I}_{\max }\right)$ for all wavelengths and $2.5 \%$ for violet as well) was examined.

Pseudomonas fluorescens and Staphylococcus epidermidis were selected as model organisms. Their choice was based on the interest to test two different species in terms of Gram-nature (a Gram-positive and a Gram-negative), but also on their relevance in several settings: hospitals, industries and households. Pseudomonas spp., indeed, are mostly studied in biofilm processes. P. fluorescens' relevance specifically stems from being the root of significant contamination problems within the food industry (dairy and meat) to agricultural microbiology. In 2010 for instance, a lot of food wastage in both Italy and Germany occurred because of $P$. fluorescens, a blue-pigment producer, which caused blue discoloration of mozzarella [60]. S. epidermidis is an opportunistic pathogen that was chosen for its large presence on skin and as well as its relevance within nosocomial biofilm-related infection [61]. Biofilms grown on a flat polystyrene surface were used to investigate the photodynamic effect. The development of biofilms on a flat surface rather than in a 96-well plate promotes the direct photon-biofilm interaction, avoiding shadow areas or regions. The choice of polystyrene (PS) was made based on the lack of specific surface chemistry to make the study general for different applications, processes and systems under control. Moreover, PS is a material of a large relevance in hospital settings, the food industry and households. The inactivation kinetics were studied by using the model of Geeraerd et al. to describe the results, estimate the inactivation parameters and make a comparison of the most effective treatments [62]. The $\log _{10}$ reductions were evaluated after each treatment and the influence of the light irradiance (inactivation by light), dosage (dose for $1-\log _{10}$ reduction) and time exposure were studied to achieve an improved understanding of the antimicrobial light-based approach. The experiments were performed on general and selective media as well, to check if the light causes sublethal injury within the biofilms. This is the first research to test five different wavelengths distributed in the visible light spectrum, each with three different irradiance levels (four for violet light), on both Gram-positive and Gram-negative biofilms. As such, this research provides a broad study of the potential of visible light as an antibiofilm treatment.

\section{Results}

Biofilms of $P$. fluorescens and S. epidermidis, grown on polystyrene, were exposed to colored LED arrays (violet, blue, green, yellow and red). Different irradiances were tested $(2.5 \%, 25 \%, 75 \%$ and $100 \%$ of $\left.I_{\max }\right)$. The Geeraerd et al. model was fitted to experimental data [62]. Specifically, for $P$. fluorescens (for all the light colors and conditions) and S. epidermidis for the violet light, the Geeraerd et al. model without shoulder was used, while for S. epidermidis for the blue, green, yellow and red light, the model without shoulder and tail, which reduced to a log-linear regression model, was employed. The model allowed for the estimation of the inactivation parameters, i.e., initial population $N_{0}$, residual population $N_{\text {res }}$ (if applicable depending on the model) and inactivation rate $k_{\max }$. Sublethal injury kinetics, expressing the sub-lethally injured cell percentages within the biofilm due to the treatment, were calculated using Equation (6) for both bacterial biofilms.

\subsection{Light Characterization}

The LED arrays were optically characterized. The emission spectra were measured, and they were found to have an emission peak $\left(\lambda_{\max }\right)$ at 400, 420, 570, 584 and $698 \mathrm{~nm}$ for violet, blue, green, yellow and red, respectively. The values of full width at half maximum, $\Delta \lambda$, ranged from 20 to $95 \mathrm{~nm}$ (details are reported in Table 1). The power densities emitted by the LED when the percentage of $I_{\max }$ was set at $2.5 \%, 25 \%, 75 \%$ and $100 \%$ are listed in Table 1 and ranged from 0.043 to $29.2 \mathrm{~mW} \mathrm{~cm}^{-2}$. After $4 \mathrm{~h}$ of light exposure, the total dose received by the biofilms was calculated using Equation (1). It ranged from 10.1 to $420.5 \mathrm{~J} \mathrm{~cm}^{-2}$ for violet, from 2.5 to $6.9 \mathrm{~J} \mathrm{~cm}^{-2}$ for blue, from 1.6 to $3.7 \mathrm{~J} \mathrm{~cm}^{-2}$ for green, from 0.6 to $2.0 \mathrm{~J} \mathrm{~cm}^{-2}$ for yellow and from 1.7 to $5.0 \mathrm{~J} \mathrm{~cm}^{-2}$ for red LED array. The violet LED array emitted 
the highest irradiance and $2.5 \% \mathrm{I}_{\max }$ was adopted to have irradiances comparable to the remaining LED arrays. The detailed irradiances and doses for each condition are also reported in Table 1.

Table 1. Light and treatments' characterization. Peak wavelengths $\left(\lambda_{\max }\right)$, values of full width at half maximum $(\Delta \lambda)$, irradiances, total doses (related to the different Light-Emitting Diodes (LED) arrays: violet, blue, green, yellow and red) and treatments.

\begin{tabular}{|c|c|c|}
\hline $\begin{array}{c}\text { Percentage } \\
\text { Maximum Irradiance }\left(I_{\max }\right)\end{array}$ & Irradiance $\left(\mathrm{mW} \mathrm{cm}^{-2}\right)$ & Total Light Dose $\left(\mathrm{J} \mathrm{cm}^{-2}\right)$ \\
\hline & \multicolumn{2}{|c|}{ Violet $\left(\lambda_{\max } / \Delta \lambda 400 / 20 \mathrm{~nm}\right)$} \\
\hline $100 \%$ & 29.2 & 420.5 \\
\hline $75 \%$ & 21 & 302.4 \\
\hline $25 \%$ & 6.9 & 99.4 \\
\hline \multirow[t]{2}{*}{$2.5 \%$} & 0.7 & 10.1 \\
\hline & \multicolumn{2}{|c|}{ Blue $\left(\lambda_{\max } / \Delta \lambda 420 / 60 \mathrm{~nm}\right)$} \\
\hline $100 \%$ & 0.49 & 6.9 \\
\hline $75 \%$ & 0.42 & 6 \\
\hline \multirow[t]{2}{*}{$25 \%$} & 0.17 & 2.5 \\
\hline & \multicolumn{2}{|c|}{ Green $\left(\lambda_{\max } / \Delta \lambda 570 / 20 \mathrm{~nm}\right)$} \\
\hline $100 \%$ & 0.26 & 3.7 \\
\hline $75 \%$ & 0.22 & 3.2 \\
\hline \multirow[t]{2}{*}{$25 \%$} & 0.11 & 1.6 \\
\hline & \multicolumn{2}{|c|}{ Yellow $\left(\lambda_{\max } / \Delta \lambda 584 / 35 \mathrm{~nm}\right)$} \\
\hline $100 \%$ & 0.14 & 2 \\
\hline $75 \%$ & 0.11 & 1.6 \\
\hline \multirow[t]{2}{*}{$25 \%$} & 0.043 & 0.6 \\
\hline & \multicolumn{2}{|c|}{$\operatorname{Red}\left(\lambda_{\max } / \Delta \lambda 698 / 95 \mathrm{~nm}\right)$} \\
\hline $100 \%$ & 0.35 & 5 \\
\hline $75 \%$ & 0.28 & 4 \\
\hline $25 \%$ & 0.11 & 1.7 \\
\hline
\end{tabular}

\subsection{Differences in Microorganisms' Biofilms}

Two model biofilms were developed from two different bacteria: P. fluorescens, which are Gram-negative bacteria, and S. epidermidis, which are Gram-positive ones. Although the mono-species biofilms were grown using the same methodology, after $24 \mathrm{~h}$ of growth, the resulting biofilms were slightly different in (i) Colony Forming Units (CFU) per area and (ii) resistance to desiccation, perhaps due to the difference in Gram classification. In fact, (i) $24 \mathrm{~h}$-grown P. fluorescens biofilms reached a population of approximately $10^{8} \mathrm{CFU} \mathrm{cm}{ }^{-2}$, while $S$. epidermidis biofilms had a population of $10^{7} \mathrm{CFU} \mathrm{\textrm {cm } ^ { - 2 }}$ (these population densities were then exposed to light treatment). Moreover, (ii) P. fluorescens biofilms were more sensitive to evaporation of water that caused some cells to die because of drying (black line and dots in Figure 1). On the contrary, S. epidermidis biofilms showed no reductions due to drying (black line and dots in Figure 2). The population of the S. epidermidis biofilm also remained constant after $4 \mathrm{~h}$.

(a)
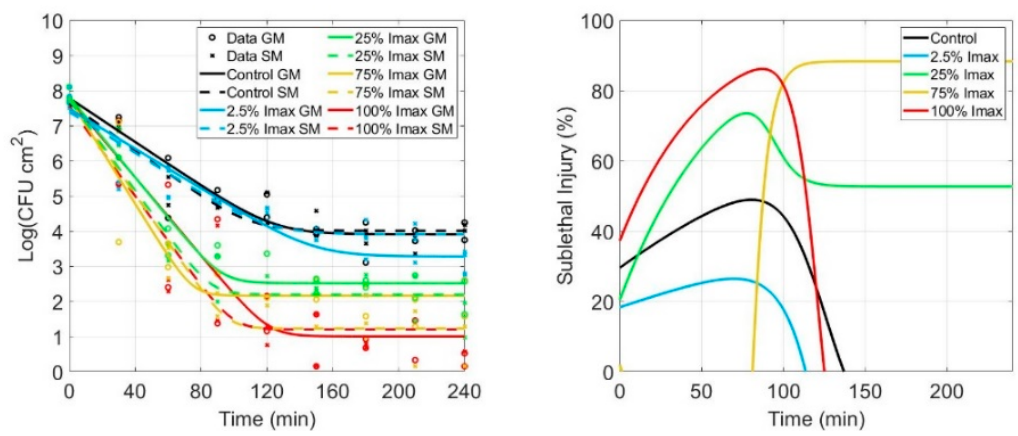

Figure 1. Cont. 
(b)

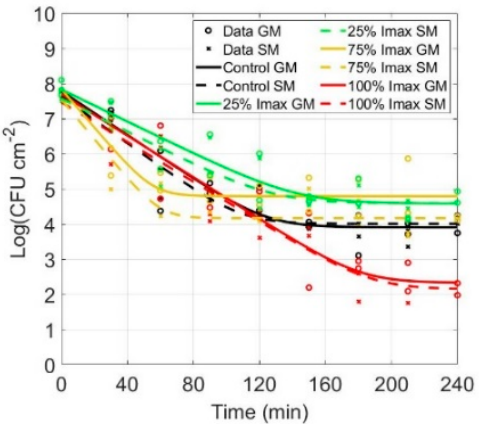

(c)

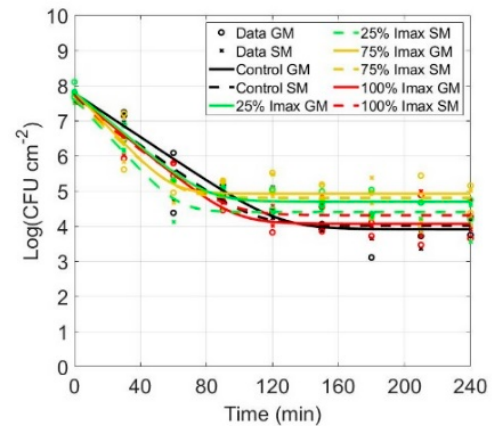

(d)

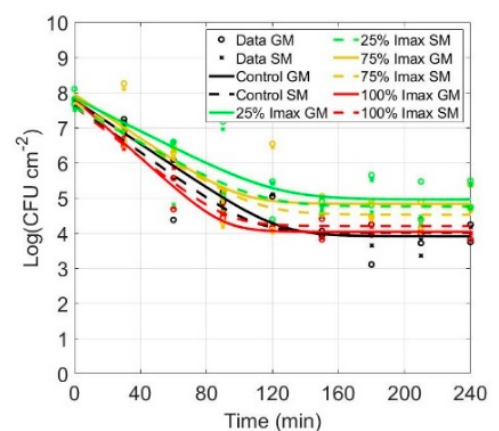

(e)

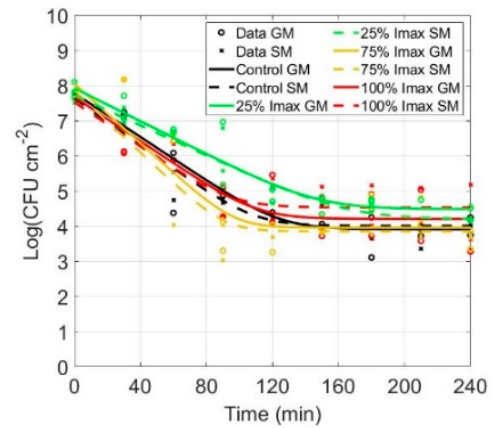

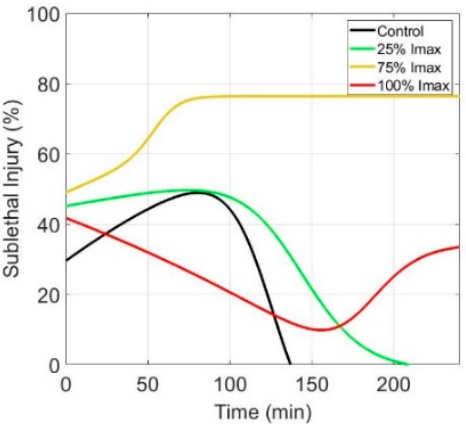
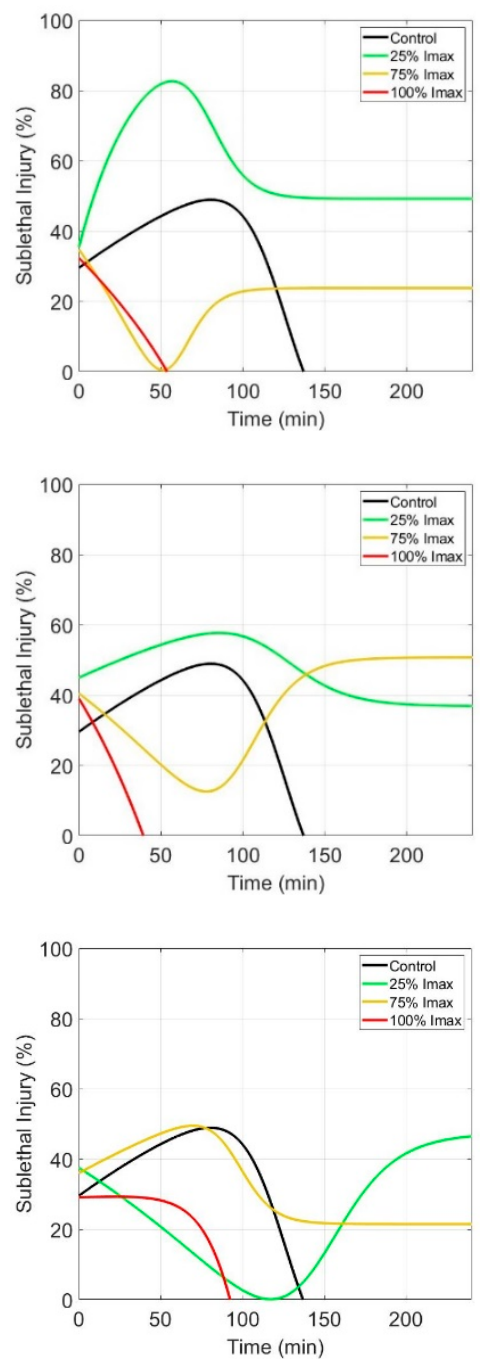

Figure 1. Inactivation and sublethal injury kinetics of $P$. fluorescens. Inactivation kinetics (first column) and sublethal injury (SI) kinetics (second column) for P. fluorescens biofilms following irradiation with (a) violet, (b) blue, (c) green, (d) yellow and (e) red LED arrays. Inactivation kinetics: the black symbols (o for general medium,GM, and $x$ for selective medium, SM) and lines (solid line for GM, dashed line for SM) represent the control (no light exposure). Likewise, the colored symbols and lines represent the fitting after $2.5 \%$ (only for violet LED array, light blue), 25\% (yellow), 75\% (green) and 100\% of $\mathrm{I}_{\max }$ (red). Sublethal injury kinetics: the colored lines represent the SI after 2.5\% (only for violet LED array, light blue), 25\% (yellow), 75\% (green) and 100\% of $\mathrm{I}_{\max }$ (red). 
(a)

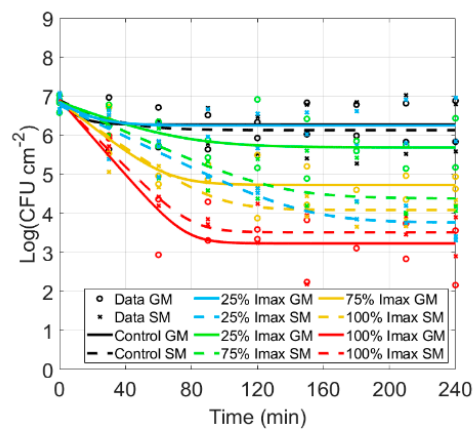

(b)

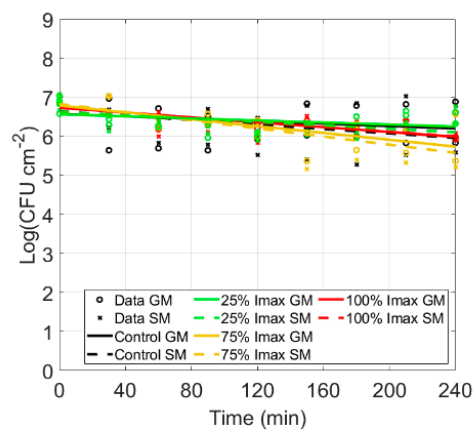

(c)

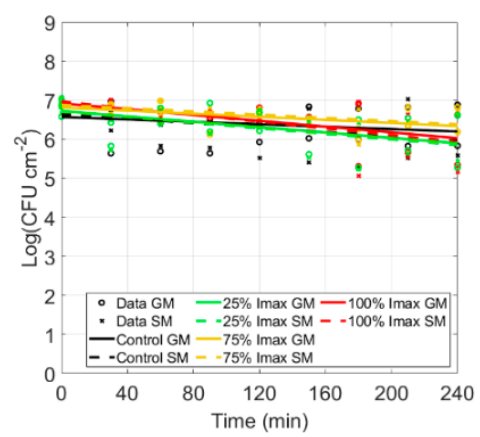

(d)

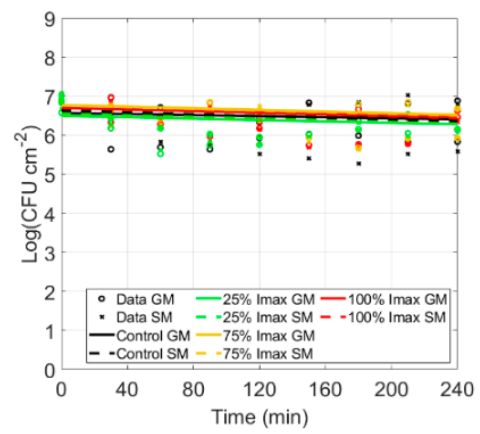

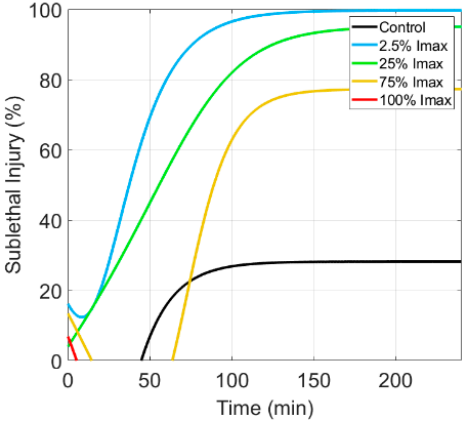
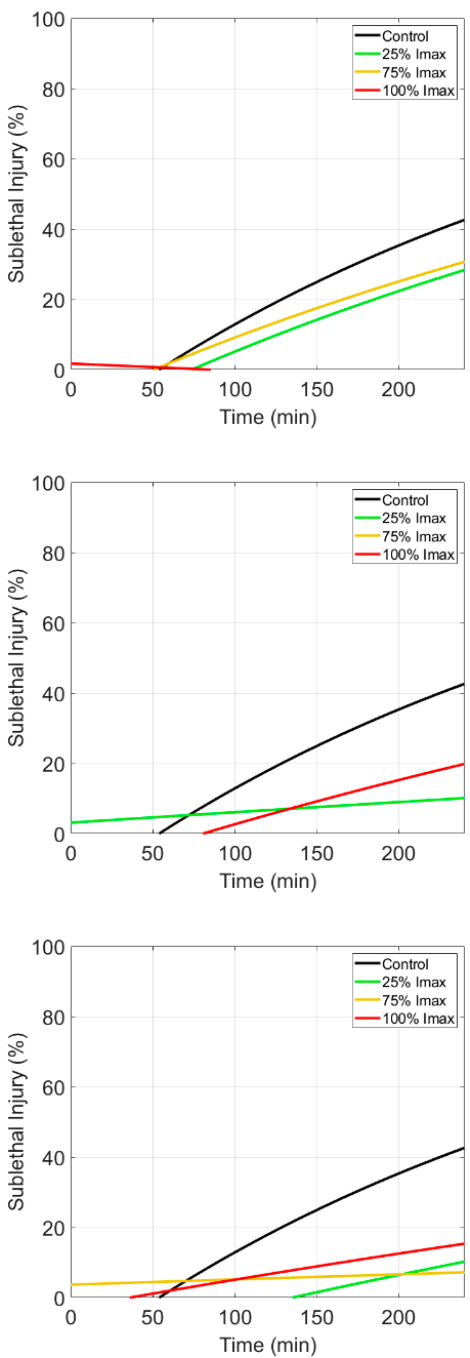

Figure 2. Cont. 
(e)

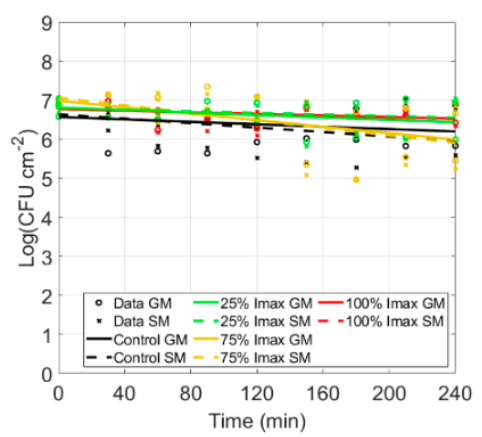

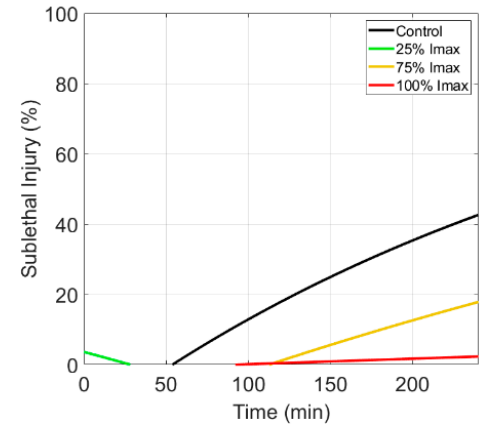

Figure 2. Inactivation and sublethal injury kinetics of S. epidermidis. Inactivation kinetics (first column) and sublethal injury (SI) kinetics (second column) for S. epidermidis biofilms following irradiation with (a) violet, (b) blue, (c) green, (d) yellow and (e) red LED arrays. Inactivation kinetics: the black symbols (o for GM, x for SM) and lines (solid line for GM, dashed line for SM) represent the control (no light exposure). Likewise, the colored symbols and lines represent the fitting after $2.5 \%$ (only for violet LED array, light blue), 25\% (yellow), 75\% (green) and 100\% of $\mathrm{I}_{\max }$ (red). Sublethal injury kinetics: the colored lines represent the SI after 2.5\% (only for violet LED array, light blue), 25\% (yellow), 75\% (green) and $100 \%$ of $\mathrm{I}_{\max }$ (red).

\subsection{P. Fluorescens}

\subsubsection{Inactivation Kinetics, Parameters and $\log _{10}$ Reductions}

The inactivation kinetics obtained by irradiating P. fluorescens biofilms utilizing violet (Figure 1a), blue (Figure 1b), green (Figure 1c), yellow (Figure 1d) and red (Figure 1e) light are presented in Figure 1. Different irradiation conditions were tested for each color $(2.5 \%$ for violet only, $25 \%, 75 \%$ and $100 \%$ of $\left.\mathrm{I}_{\max }\right) . N_{0}, N_{\text {res }}$ and $k_{\max }$ were estimated and their respective standard errors (SE) and the root mean squared error (RMSE) values were calculated. These values were summarized in Table 2, along with the statistical analysis. The overall $\log _{10}$ reductions $\left(N_{0}-N_{\text {res }}\right)$ were also calculated and are listed in the same table.

The inactivation kinetics are characterized by a log-linear inactivation phase, from time 0 to approximately $90-100 \mathrm{~min}$, followed by a tailing phase (Figure 1). The log-linear inactivation is present in the control as well, due to a desiccation process occurring to the biofilms within the first $100 \mathrm{~min}$.

Regardless of the treatment, the rate of inactivation $k_{\max }$ was in general very similar to the control (no significant differences), as can be noticed from the $k_{\max }$ values displayed in Table 2. This means that the inactivation rate was not influenced by the light exposure, while the residual population $N_{\text {res }}$ was strongly influenced in some conditions.

Different percentages of $\mathrm{I}_{\max }\left(2.5 \%\right.$ for violet only, $25 \%, 75 \%$ and $100 \%$ of $\left.\mathrm{I}_{\max }\right)$ per LED color resulted in higher or equivalent $\log _{10}$ reductions compared to the control (no light exposure). The $\log _{10}$ reductions indicated that the highest reductions were obtained with violet light (in all conditions) and blue light when $\mathrm{I}_{\max }$ equaled $100 \%$. Indeed, the most effective inactivation was observed when the violet

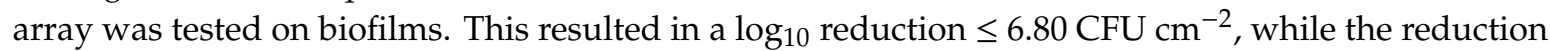
of the control was only $3.87 \mathrm{CFU} \mathrm{cm}{ }^{-2}$ (due to natural desiccation). A $\log _{10}$ reduction $\leq 5.37 \mathrm{CFU} \mathrm{cm}{ }^{-2}$ was induced for biofilms exposed to blue LED irradiation. Furthermore, experiments were carried out with green, yellow and red irradiation. These revealed no additional reduction compared to the control. 
Table 2. P. fluorescens parameters. Estimated parameters with standard errors of $P$. fluorescens inactivation using the Geeraerd et al. model are listed, i.e., $N_{0}$, $N_{\text {res }}, k_{\text {max }}$,

$\log _{10}$ reduction and root mean squared error (RMSE) for general and selective media [62].

\begin{tabular}{|c|c|c|c|c|c|c|c|c|c|c|c|}
\hline \multirow[b]{2}{*}{ LED } & \multirow[b]{2}{*}{$\begin{array}{l}I_{\max } \\
(\%)\end{array}$} & \multicolumn{5}{|c|}{ General Medium } & \multicolumn{5}{|c|}{ Selective Medium } \\
\hline & & $\begin{array}{c}\log _{10} N_{0} \\
(\log (C F U \\
\left.\left.\mathrm{cm}^{-2}\right)\right)\end{array}$ & $\begin{array}{c}\log _{10} N_{r e s} \\
(\log (C F U \\
\left.\left.\mathrm{cm}^{-2}\right)\right)\end{array}$ & $k_{\max }\left(\min ^{-1}\right)$ & $\begin{array}{c}\log _{10} \\
\text { Reduction } \\
\text { (Log(CFU } \\
\left.\mathrm{cm}^{-2}\right) \text { ) }\end{array}$ & RMSE & $\begin{array}{c}\log _{10} N_{0} \\
(\log (C F U \\
\left.\left.\mathrm{cm}^{-2}\right)\right)\end{array}$ & $\begin{array}{c}\log _{10} N_{\text {res }} \\
\left(\log \left(\mathrm{CFU} \mathrm{cm}^{-2}\right)\right)\end{array}$ & $k_{\max }\left(\min ^{-1}\right)$ & $\begin{array}{c}\log _{10} \\
\text { Reduction } \\
(\log (\mathrm{CFU} \\
\left.\left.\mathrm{cm}^{-2}\right)\right)\end{array}$ & RMSI \\
\hline Control & 0 & a $7.781 \pm 0.200$ & a $3.907 \pm 0.182$ & a $0.072 \pm 0.009$ & a $3.873 \pm 0.270$ & 0.4926 & a $7.629 \pm 0.170$ & a $4.006 \pm 0.142$ & a $0.077 \pm 0.009$ & $\mathrm{a}, \mathrm{e} 3.623 \pm 0.222$ & 0.4123 \\
\hline Violet & $\begin{array}{c}2.5 \\
25 \\
75 \\
100 \\
\end{array}$ & $\begin{array}{l}\text { a } 7.478 \pm 0.208 \\
\text { a } 7.823 \pm 0.215 a \\
\text { a } 7.778 \pm 0.311_{a} \\
\text { a } 7.804 \pm 0.394_{a}\end{array}$ & $\begin{array}{l}\text { b } 3.275 \pm 0.222 \\
\text { c } 2.517 \pm 0.170_{a} \\
\text { c } 2.158 \pm 0.221_{a} \\
\text { d } 1.000 \pm 0.329_{a}\end{array}$ & $\begin{array}{l}\mathrm{a} 0.065 \pm 0.007 \\
\text { a } 0.132 \pm 0.013 \mathrm{a} \\
{ }^{\mathrm{a}} 0.173 \pm 0.024 \mathrm{a} \\
\mathrm{a} 0.134 \pm 0.018 \mathrm{a}\end{array}$ & $\begin{array}{l}\text { a } 4.203 \pm 0.304 \\
\text { c } 5.307 \pm 0.274 \\
\text { c } 5.619 \pm 0.382 \\
\text { b } 6.804 \pm 0.513\end{array}$ & $\begin{array}{l}0.5263 \\
0.5125 \\
0.7229 \\
0.9002 \\
\end{array}$ & $\begin{array}{l}\text { a } 7.562 \pm 0.424 \\
\text { a } 7.769 \pm 0.272_{a} \\
\text { a } 7.724 \pm 0.245_{a} \\
\text { a } 7.390 \pm 0.176_{a}\end{array}$ & $\begin{array}{l}\text { a } 1.200 \pm 0.325 \\
\text { c } 1.227 \pm 0.217 \mathrm{a} \\
\text { b } 2.192 \pm 0.186_{a} \\
\text { b } 3.911 \pm 0.157 \mathrm{a}\end{array}$ & $\begin{array}{l}\text { a } 0.148 \pm 0.023 \\
\text { a } 0.157 \pm 0.015 a \\
\text { a } 0.148 \pm 0.017 \text { a } \\
\text { a } 0.067 \pm 0.008 \text { a }\end{array}$ & $\begin{array}{l}\text { a } 3.479 \pm 0.236 \\
\text { c } 5.532 \pm 0.307 \\
\text { b } 6.543 \pm 0.348 \\
\text { b } 6.362 \pm 0.534\end{array}$ & $\begin{array}{l}0.4311 \\
0.5752 \\
0.6525 \\
0.9407 \\
\end{array}$ \\
\hline Blue & $\begin{array}{c}25 \\
75 \\
100 \\
\end{array}$ & $\begin{array}{l}\text { a } 7.828 \pm 0.182 a \\
\text { a } 7.838 \pm 0.232 a \\
\text { a } 7.701 \pm 0.237 a\end{array}$ & $\begin{array}{l}\mathrm{b} 4.583 \pm 0.195_{\mathrm{b}} \\
\mathrm{b} 4.794 \pm 0.150_{\mathrm{b}} \\
\mathrm{c} 2.327 \pm 0.327_{\mathrm{b}}\end{array}$ & $\begin{array}{l}\text { a } 0.053 \pm 0.007 \mathrm{a} \\
\text { a } 0.126 \pm 0.028 \mathrm{a} \\
\text { a } 0.068 \pm 0.007 \mathrm{a}\end{array}$ & $\begin{array}{l}\text { a,c } 3.245 \pm 0.266 \\
\text { c } 3.044 \pm 0.277 \\
\text { b } 5.374 \pm 0.404 \\
\end{array}$ & $\begin{array}{l}0.4926 \\
0.5279 \\
0.6217 \\
\end{array}$ & $\begin{array}{l}\text { a } 7.467 \pm 0.264^{\mathrm{a}} \\
\text { a } 7.545 \pm 0.225_{a} \\
\text { a } 7.567 \pm 0.198_{a}\end{array}$ & $\begin{array}{c}\mathrm{c}^{\mathrm{c}} 2.144 \pm 0.391_{\mathrm{b}} \\
\mathrm{a}, \mathrm{c} 4.167 \pm 0.148_{\mathrm{b}, \mathrm{c}} \\
\mathrm{b} 4.591 \pm 0.191_{\mathrm{b}}\end{array}$ & $\begin{array}{l}\text { a } 0.065 \pm 0.007 a \\
\text { a } 0.130 \pm 0.024 a \\
\text { a } 0.054 \pm 0.009 a\end{array}$ & $\begin{array}{l}\text { a } 2.977 \pm 0.275 \\
\text { a } 3.378 \pm 0.269 \\
\text { b } 5.323 \pm 0.472\end{array}$ & $\begin{array}{l}0.4879 \\
0.5142 \\
0.6978 \\
\end{array}$ \\
\hline Green & $\begin{array}{c}25 \\
75 \\
100 \\
\end{array}$ & $\begin{array}{l}\text { a } 7.808 \pm 0.109 a \\
\text { a } 7.820 \pm 0.207 a \\
\text { a } 7.724 \pm 0.158 a\end{array}$ & $\begin{array}{l}\mathrm{b} 4.693 \pm 0.078_{\mathrm{b}} \\
\mathrm{b} 4.920 \pm 0.136_{\mathrm{c}} \\
\mathrm{a} 4.063 \pm 0.123_{\mathrm{c}}\end{array}$ & $\begin{array}{l}\text { a } 0.090 \pm 0.009 a \\
\text { a } 0.113 \pm 0.023 a \\
\text { a } 0.087 \pm 0.009 a\end{array}$ & $\begin{array}{c}\mathrm{b}, \mathrm{c} 3.115 \pm 0.134 \\
\mathrm{c} 2.900 \pm 0.248 \\
\mathrm{a}, \mathrm{b} 3.660 \pm 0.200\end{array}$ & $\begin{array}{l}0.2531 \\
0.4726 \\
0.3775 \\
\end{array}$ & $\begin{array}{l}\text { a } 7.554 \pm 0.155_{a} \\
\text { a } 7.635 \pm 0.218 a \\
\text { a } 7.618 \pm 0.151_{a}\end{array}$ & $\begin{array}{c}\mathrm{a}, \mathrm{b} 4.304 \pm 0.120_{\mathrm{b}} \\
\mathrm{b} 4.802 \pm 0.147_{\mathrm{c}} \\
\mathrm{a}, \mathrm{b} 4.399 \pm 0.101_{\mathrm{c}}\end{array}$ & $\begin{array}{l}\mathrm{a} 0.080 \pm 0.010_{\mathrm{a}} \\
\mathrm{a} 0.103 \pm 0.022_{\mathrm{a}} \\
\mathrm{a} 0.118 \pm 0.015_{\mathrm{a}}\end{array}$ & $\begin{array}{c}\mathrm{a}, \mathrm{b} 3.219 \pm 0.181 \\
\mathrm{~b} 2.833 \pm 0.263 \\
\mathrm{a}, \mathrm{b} 3.251 \pm 0.196\end{array}$ & $\begin{array}{l}0.3462 \\
0.5016 \\
0.3680 \\
\end{array}$ \\
\hline Yellow & $\begin{array}{c}25 \\
75 \\
100\end{array}$ & $\begin{array}{l}\text { a } 7.841 \pm 0.223 a \\
\text { a } 7.950 \pm 0.268 \mathrm{a} \\
\text { a } 7.818 \pm 0.104 a\end{array}$ & $\begin{array}{l}\mathrm{b} 4.954 \pm 0.210_{\mathrm{b}} \\
\mathrm{b} 4.828 \pm 0.215_{\mathrm{c}} \\
\mathrm{a} 4.033 \pm 0.082_{\mathrm{c}}\end{array}$ & $\begin{array}{l}\text { a } 0.054 \pm 0.010 a \\
\text { a } 0.072 \pm 0.015 a \\
\text { a } 0.097 \pm 0.007 a\end{array}$ & $\begin{array}{l}\mathrm{b} 2.888 \pm 0.306 \\
\mathrm{~b} 3.122 \pm 0.344 \\
\mathrm{a} 3.785 \pm 0.132\end{array}$ & $\begin{array}{l}0.5457 \\
0.6421 \\
0.2453\end{array}$ & $\begin{array}{l}\text { a } 7.604 \pm 0.105 a \\
\text { a } 7.725 \pm 0.267 a \\
\text { a } 7.582 \pm 0.257 a\end{array}$ & $\begin{array}{c}\mathrm{b} 4.197 \pm 0.081_{\mathrm{b}} \\
\mathrm{a}, \mathrm{b} 4.508 \pm 0.245_{\mathrm{c}} \\
\mathrm{a} 4.754 \pm 0.226_{\mathrm{c}}\end{array}$ & $\begin{array}{l}\text { a } 0.084 \pm 0.006_{a} \\
\text { a } 0.066 \pm 0.014_{a} \\
\text { a } 0.058 \pm 0.013_{a}\end{array}$ & 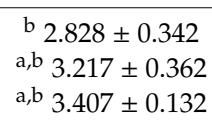 & $\begin{array}{l}0.6230 \\
0.6478 \\
0.2494\end{array}$ \\
\hline
\end{tabular}

$\mathrm{a}, \mathrm{b}, \mathrm{c}, \mathrm{d}$ Effect of the color: For each percentage of $\mathrm{I}_{\max }$ estimated parameters bearing different subscripts following the values (no letter in common) are significantly different $(p \leq 0.05)$.

Effect of the percentage of $I_{\max }$ : For each color, estimated parameters bearing different superscripts in front of the values (no letter in common) are significantly different $(p \leq 0.05)$. 


\subsubsection{Sublethal Injury}

The model was also fitted to the data resulting from the selective plating media. The inactivation kinetics (dashed lines) and the parameters, related to selective media and estimated from the Geeraerd et al. [62] model, are displayed in Figure 1 and Table 2. The sublethal injury of P. fluorescens was determined by plating the bacterial suspension on general (GM) and selective media (SM) to quantify the sublethal cell damage after irradiation. Preliminary experiments were carried out to check that SM did not affect the growth of the cells of the biofilms after mechanical detachment of the biofilm using a scraper. The SI curve related to the control experiments (no light exposure) showed a peak at about $50 \%$. This corresponds to the phase-change between the log-linear phase and the tail in the inactivation curve (column 1, Figure 1). As violet exposure is tested, SI curves have a higher peak corresponding to $25 \%$ and $100 \% \mathrm{I}_{\max }$ and a lower one corresponding to $2.5 \% \mathrm{I}_{\max }$ in the same time frame. $75 \% \mathrm{I}_{\max }$ is an exception, showing a plateau corresponding to the tail of the inactivation curve. Generally, the other SI curves have a similar trend compared to the control, except for $75 \% \mathrm{I}_{\max }$ with blue and green exposure.

\subsection{S. Epidermidis}

\subsubsection{Inactivation Kinetics, Parameters and $\log _{10}$ Reduction}

For S. epidermidis biofilms, similar experiments were carried out. Figure 2 displays the inactivation kinetics obtained using violet (Figure 2a), blue (Figure 2b), green (Figure 2c), yellow (Figure 2d) and red (Figure 2e) LED arrays and the models fitting the data. The estimated parameters $N_{0}, N_{\text {res }}$ (if applicable depending on the model) and $k_{\max }$, the respective SE, RMSE values and the $\log _{10}$ reduction are summarized in Table 3, for both models. As in the previous P. fluorescens experiment, different irradiation conditions ( $2.5 \%$ for violet only, $25 \%, 75 \%$ and $100 \%$ of $\mathrm{I}_{\max }$ ) were tested for each color.

For biofilms exposed to violet light, the inactivation kinetics were characterized by a log-linear inactivation phase, lasting approximately 80 to $90 \mathrm{~min}$, after which a tailing phase followed (Figure 2). The control did not undergo a desiccation process as seen with $P$. fluorescens. The initial population was approximately $6.84 \mathrm{CFU} \mathrm{cm}{ }^{-2}$ and the residual population was almost the same $\left(6.27 \mathrm{CFU} \mathrm{cm} \mathrm{cm}^{-2}\right)$. The standard errors of the inactivation rate, $k_{\max }$, demonstrate that this parameter could not be estimated accurately. Based on the parameters' estimation, the $\log _{10}$ reduction was determined and had a much better accuracy. When blue, green, yellow and red LED arrays were used (in all conditions), the cells within the biofilm after $4 \mathrm{~h}$ of treatment were almost the same compared to the control, and the model without shoulder and without tail (log-linear regression) was used to fit the data and represent the kinetics. The rates of inactivation, $k_{\max }$, did not show any increase for these LED array exposures (Table 3 ).

A $\log _{10}$ reduction of approximately $3.69 \mathrm{CFU} \mathrm{cm} \mathrm{cm}^{-2}$ was induced by violet exposure (harshest condition) compared to approximately $0.57 \mathrm{CFU} \mathrm{cm} \mathrm{c}^{-2}$ of the control. Regarding biofilms exposed to other colors (blue, green, yellow and red), no substantial reduction was observed in terms of biofilm populations (Figure 2). The estimated reductions for each condition are reported in detail for both general and selective media in Table 3.

\subsubsection{Sublethal Injury}

The different evolution in SI for controls of S. epidermidis for violet light (Figure 2a) and blue, yellow, green and red light (Figure $2 b-e$ ) were caused by the use of two different (simplified) versions of the Geeraerd et al. model (as explained in Section 4.7). With regards to SI kinetics related to violet light, the maximum SI value decreases $(100 \%, 95 \%$ and $75 \%$ related to the plateau values) as the irradiance increases from $2.5 \%$ to $75 \% \mathrm{I}_{\max }$ (Figure 2). This means that the stronger the irradiance, the stronger the killing effect. Indeed, in the harshest condition, the majority of the cells are dead rather than damaged. No evidence of this trend is present when $\mathrm{I}_{\max }$ equals $100 \%$. Instead, SI curves lay underneath the control for blue, green, yellow and red exposures. Negligible SI values were observed for those treatments. 
Table 3. S. epidermidis parameters. Estimated parameters with standard errors of S. epidermidis inactivation using the Geeraerd et al. model are listed, i.e., $N_{0}$, $N_{\text {res }}, k_{m a x}$ $\log _{10}$ reduction and RMSE for general and selective media [62].

\begin{tabular}{|c|c|c|c|c|c|c|c|c|c|c|c|}
\hline \multirow[b]{3}{*}{ LED } & \multirow[b]{3}{*}{$I_{\max }(\%)$} & \multicolumn{10}{|c|}{ Geeraerd et al. Model [62] } \\
\hline & & \multicolumn{5}{|c|}{ General Medium } & \multicolumn{5}{|c|}{ Selective Medium } \\
\hline & & $\begin{array}{c}\log _{10} N_{0} \\
(\log (C F U \\
\left.\left.\mathrm{cm}^{-2}\right)\right)\end{array}$ & $\begin{array}{c}\log _{10} N_{\text {res }} \\
(\log (\mathrm{CFU} \\
\left.\left.\mathrm{cm}^{-2}\right)\right)\end{array}$ & $k_{\max }\left(\min ^{-1}\right)$ & $\begin{array}{c}\log _{10} \text { Reduction } \\
(\log (\mathrm{CFU} \\
\left.\left.\mathrm{cm}^{-2}\right)\right)\end{array}$ & RMSE & $\begin{array}{c}\log _{10} N_{0} \\
(\log (C F U \\
\left.\left.\mathrm{cm}^{-2}\right)\right)\end{array}$ & $\begin{array}{c}\log _{10} N_{r e s} \\
(\log (C F U \\
\left.\left.\mathrm{cm}^{-2}\right)\right)\end{array}$ & $k_{\max }\left(\min ^{-1}\right)$ & $\begin{array}{c}\log _{10} \text { Reduction } \\
(\log (\text { CFU } \\
\left.\left.\mathrm{cm}^{-2}\right)\right)\end{array}$ & RMSE \\
\hline Control & 0 & a $6.844 \pm 0.208$ & a $6.275 \pm 0.125$ & a $0.125 \pm 0.446$ & ${ }^{\mathrm{a}} 0.569 \pm 0.242$ & 0.4645 & a $6.900 \pm 0.244$ & a $6.132 \pm 0.162$ & a $0.056 \pm 0.060$ & a $0.768 \pm 0.293$ & 0.5486 \\
\hline \multirow{6}{*}{ Violet } & 2.5 & a $6.847 \pm 0.180$ & a $6.254 \pm 0.112$ & a $0.076 \pm 0.100$ & a $0.593 \pm 0.212$ & 0.3408 & a $6.771 \pm 0.135$ & b $3.762 \pm 0.157$ & a $0.046 \pm 0.005$ & $\mathrm{~b}, \mathrm{c} 3.008 \pm 0.207$ & 0.4023 \\
\hline & 25 & $\mathrm{a} 6.859 \pm 0.219 \mathrm{a}$ & b $5.687 \pm 0.179$ & a $0.038 \pm 0.021_{a}$ & a $1.172 \pm 0.283$ & 0.5097 & a $6.842 \pm 0.209 a$ & c $4.382 \pm 0.219$ & a $0.043 \pm 0.009$ a & b $2.460 \pm 0.303$ & 0.5182 \\
\hline & 75 & a $6.879 \pm 0.193_{a}$ & ${ }^{c} 4.729 \pm 0.140$ & a $0.076 \pm 0.019 a$ & b $2.149 \pm 0.238$ & 0.4441 & a $6.816 \pm 0.164_{a}$ & $\mathrm{~b}, \mathrm{c} 4.086 \pm 0.143$ & a $0.066 \pm 0.010_{a}$ & b $2.730 \pm 0.217$ & 0.3894 \\
\hline & 100 & a $6.923 \pm 0.258 \mathrm{a}$ & d $3.232 \pm 0.189$ & a $0.111 \pm 0.020_{\mathrm{a}}$ & c $3.690 \pm 0.320$ & 0.6000 & a $6.892 \pm 0.193$ a & b $3.516 \pm 0.145$ & a $0.098 \pm 0.014_{\mathrm{a}}$ & c $3.376 \pm 0.293$ & 0.4500 \\
\hline & & \multicolumn{10}{|c|}{ Geeraerd et al. Model, Reduced to Log-Linear Regression } \\
\hline & & \multicolumn{5}{|c|}{ General Medium } & \multicolumn{5}{|c|}{ Selective Medium } \\
\hline LED & $I_{\max }(\%)$ & $\log _{10} N_{0}(\log$ & $\left(\mathrm{CFU} \mathrm{\textrm {cm } ^ { - 2 } ) )}\right.$ & $k_{\max }$ & $\left.\min ^{-1}\right)$ & RMSE & $\log _{10} N_{0}(\mathrm{~L}$ & $\left(\mathrm{CFU} \mathrm{\textrm {cm } ^ { - 2 } ) )}\right.$ & \multicolumn{2}{|c|}{$k_{\max }\left(\min ^{-2}\right)$} & RMSE \\
\hline Control & 0 & \multicolumn{2}{|c|}{${ }^{a} 6.574 \pm 0.174$} & \multicolumn{2}{|c|}{ a $1.5 \times 10^{-3} \pm 1.3 \times 10^{-3}$} & 0.5002 & \multicolumn{2}{|c|}{${ }^{\mathrm{a}} 6.674 \pm 0.201$} & \multicolumn{2}{|c|}{ a $2.8 \times 10^{-3} \pm 1.5 \times 10^{-3}$} & 0.5786 \\
\hline \multirow{3}{*}{ Blue } & 25 & \multirow{3}{*}{\multicolumn{2}{|c|}{$\begin{array}{l}\mathrm{a} 6.787 \pm 0.123_{\mathrm{a}} \\
\mathrm{a} 6.735 \pm 0.058_{\mathrm{a}}\end{array}$}} & \multirow{3}{*}{\multicolumn{2}{|c|}{$\begin{array}{l}\text { a } 1.3 \times 10^{-3} \pm 7.0 \times 10^{-4}{ }_{a} \\
\text { a } 4.4 \times 10^{-3} \pm 9.0 \times 10^{-4}{ }^{a} \\
\text { a } 3.1 \times 10^{-3} \pm 4.0 \times 10^{-4}{ }_{a}\end{array}$}} & 0.2963 & \multirow{3}{*}{\multicolumn{2}{|c|}{$\begin{array}{l}\text { a } 6.628 \pm 0.104 a \\
\text { a } 6.830 \pm 0.140 \mathrm{a} \\
\text { a } 6.735 \pm 0.093_{a}\end{array}$}} & \multirow{3}{*}{\multicolumn{2}{|c|}{$\begin{array}{l}\text { a } 2.2 \times 10^{-3} \pm 8.0 \times 10^{-4}{ }_{a} \\
\text { a } 5.2 \times 10^{-3} \pm 1.1 \times 10^{-3}{ }^{a} \\
\text { a } 3.0 \times 10^{-3} \pm 7.0 \times 10^{-4}{ }_{a}\end{array}$}} & 0.3110 \\
\hline & 75 & & & & & 0.3534 & & & & & 0.4019 \\
\hline & 100 & & & & & 0.2568 & & & & & 0.1671 \\
\hline \multirow{3}{*}{ Green } & 25 & \multirow{3}{*}{\multicolumn{2}{|c|}{$\begin{array}{l}\text { a } 6.728 \pm 0.159_{a} \\
\text { a } 6.815 \pm 0.091_{a} \\
\text { a } 6.922 \pm 0.139_{a}\end{array}$}} & \multirow{3}{*}{\multicolumn{2}{|c|}{$\begin{array}{l}\text { a } 3.5 \times 10^{-3} \pm 1.2 \times 10^{-3}{ }^{a} \\
\text { a } 2.0 \times 10^{-3} \pm 7.0 \times 10^{-4}{ }^{a} \\
\text { a } 3.7 \times 10^{-3} \pm 1.1 \times 10^{-3}{ }_{a}\end{array}$}} & 0.4632 & & \multirow{3}{*}{\multicolumn{2}{|c|}{$\begin{array}{l}\text { a } 3.6 \times 10^{-3} \pm 1.2 \times 10^{-3}{ }^{a} \\
\text { a } 2.1 \times 10^{-3} \pm 7.0 \times 10^{-4}{ }_{a} \\
\text { a } 4.3 \times 10^{-3} \pm 1.2 \times 10^{-3}{ }_{a}\end{array}$}} & 0.4586 \\
\hline & 75 & & & & & 0.2769 & a 6.882 & $=0.096_{\mathrm{a}}$ & & & 0.2627 \\
\hline & 100 & & & & & 0.4645 & a 6.970 & $=0.161_{\mathrm{a}}$ & & & 0.4008 \\
\hline \multirow{3}{*}{ Yellow } & 25 & \multirow{3}{*}{\multicolumn{2}{|c|}{$\begin{array}{l}\text { a } 6.499 \pm 0.132_{a} \\
\text { a } 6.779 \pm 0.112_{a} \\
\text { a } 6.703 \pm 0.122_{a}\end{array}$}} & \multirow{3}{*}{\multicolumn{2}{|c|}{$\begin{array}{l}\text { a } 2.0 \times 10^{-3} \pm 1.0 \times 10^{-3}{ }^{a} \\
\text { a } 2.4 \times 10^{-3} \pm 8.0 \times 10^{-4}{ }_{\text {a }} \\
\text { a } 2.3 \times 10^{-3} \pm 9.0 \times 10^{-4}{ }_{\text {a }}\end{array}$}} & 0.3632 & \multirow{3}{*}{\multicolumn{2}{|c|}{$\begin{array}{l}\text { a } 6.559 \pm 0.126_{a} \\
\text { a } 6.763 \pm 0.117 \mathrm{a} \\
\text { a } 6.716 \pm 0.121_{a}\end{array}$}} & \multirow{3}{*}{\multicolumn{2}{|c|}{$\begin{array}{l}\text { a } 2.5 \times 10^{-3} \pm 9.0 \times 10^{-4}{ }_{a} \\
\text { a } 2.5 \times 10^{-3} \pm 8.0 \times 10^{-4}{ }_{a} \\
\text { a } 2.7 \times 10^{-3} \pm 9.0 \times 10^{-4}{ }_{a}\end{array}$}} & 0.3798 \\
\hline & 75 & & & & & 0.3374 & & & & & 0.3222 \\
\hline & 100 & & & & & 0.3497 & & & & & 0.3505 \\
\hline \multirow{3}{*}{ Red } & 25 & \multirow{3}{*}{\multicolumn{2}{|c|}{$\begin{array}{l}\text { a } 6.812 \pm 0.120_{a} \\
\text { a } 6.989 \pm 0.195 a \\
\text { a } 6.772 \pm 0.081_{a}\end{array}$}} & & & 0.3478 & a 6.796 & $=0.121_{a}$ & a $1.0 \times 10^{-3}$ & $=9.0 \times 10^{-4}{ }_{a}$ & 0.345 \\
\hline & 75 & & & a $4.1 \times 10^{-3}$ & $\pm 1.5 \times 10^{-3} a$ & 0.6212 & a 7.064 & $=0.219 \mathrm{a}$ & a $4.8 \times 10^{-3}$ & $=1.7 \times 10^{-3}{ }_{a}$ & 0.5626 \\
\hline & 100 & & & a $1.0 \times 10^{-}$ & $\pm 6.0 \times 10^{-4}{ }_{a}^{a}$ & 0.3034 & a 6.778 & $=0.105_{\mathrm{a}}$ & a $1.1 \times 10^{-3}$ & $=7.0 \times 10^{-4}{ }_{a}^{a}$ & 0.2341 \\
\hline
\end{tabular}

$\mathrm{a}, \mathrm{b}, \mathrm{c}, \mathrm{d}$ Effect of the color: For each percentage of $\mathrm{I}_{\max }$, estimated parameters bearing different superscripts following the values (no letter in common) are significantly different $(p \leq 0.05)$.

Effect of the percentage of $\mathrm{I}_{\max }$ : For each color, estimated parameters bearing different subscripts in front of the values (no letter in common) are significantly different $(p \leq 0.05)$. 


\subsection{Inactivation by Light and Dose for 1- $\log _{10}$ Reduction}

To understand the mere effect of the irradiation on biofilms, without considering the effect of the desiccation that results in a reduction of the population of P. fluorescens (Section 3.2), the inactivation by light itself was calculated $\left(N_{\text {res }}\right.$ control $-N_{\text {res }}$ light). This data analysis concerns the fits obtained with violet-exposed biofilms, for which the residual population was estimated through the Geeraerd et al. model [62]. In Figure 3, the population of bacteria killed employing violet light exposure $\left(N_{\text {res }}\right.$ control $-N_{\text {res }}$ light) was reported as a function of the percentage of $\mathrm{I}_{\max }$. Figure 3 demonstrates that light treatments with $100 \%$ and $75 \% \mathrm{I}_{\max }$ were found to cause a reduction of approximately $3.0 \log _{10}$ and $1.5 \log _{10}$ CFU cm$~^{-2}$ in the population of both P. fluorescens and S. epidermidis biofilms. Whereas $25 \%$ and $2.5 \%$ $\mathrm{I}_{\max }$ caused a reduction of approximately 1.39 and $0.63 \log _{10} \mathrm{CFU} \mathrm{cm} \mathrm{cm}^{-2}$ for P. fluorescens respectively, and for S. epidermidis, 0.59 and $0.02 \log _{10} \mathrm{CFU} \mathrm{cm}{ }^{-2}$, respectively.

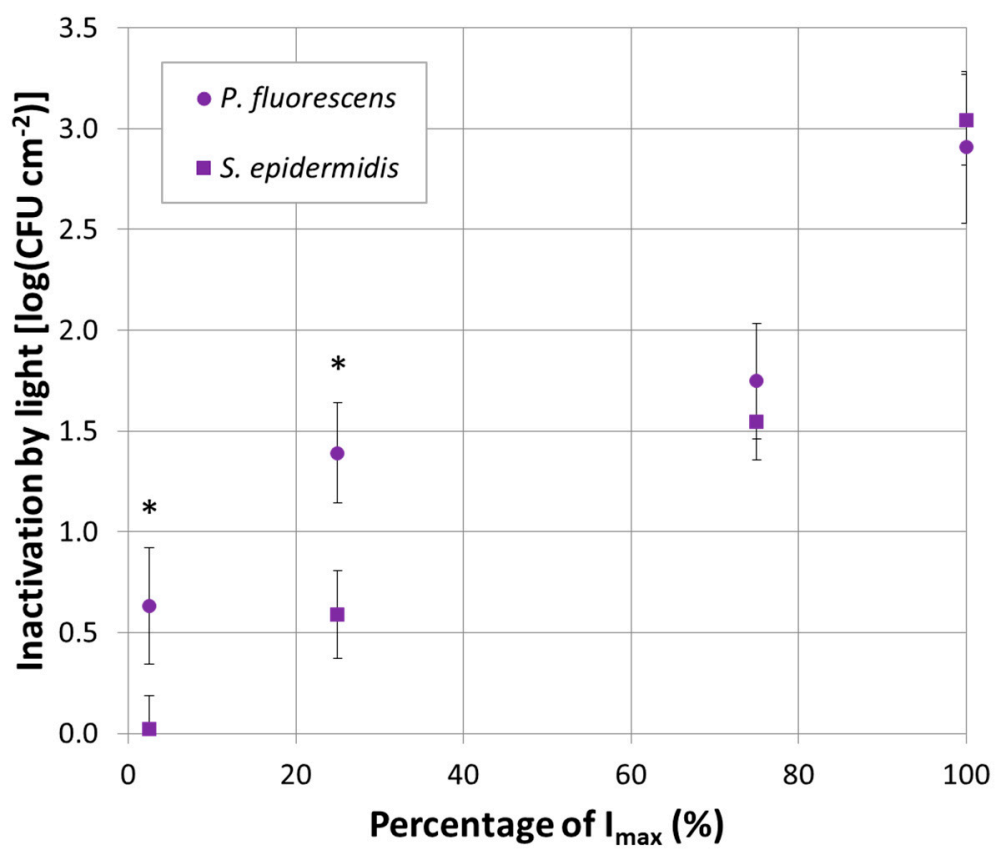

Figure 3. Violet LED treatments. Inactivation by light of P. fluorescens (round symbols) and S. epidermidis (squared symbols) under violet exposure with $2.5 \%, 25 \%, 75 \%$ and $100 \%$ of $\mathrm{I}_{\max }$. The ${ }^{*}$ symbol indicates significant differences $(p<0.05)$.

Moreover, the dose for 1- $\log _{10}$ reduction was calculated following Equation (6). It represents the required dose to produce $1-\log _{10}$ inactivation in the population of the specific bacterial strain. It was estimated for each violet-investigated condition. It is displayed for both P. fluorescens and S. epidermidis as a function of the irradiance (Figure 4). The most effective treatments (violet treatments and $100 \%$ $\mathrm{I}_{\max }$ with blue light on P. fluorescens) were considered in this analysis.

Figure 4 reveals that the dose required for $1-\log _{10}$ reduction was $\geq 31 \mathrm{~J} \mathrm{~cm}^{-2}$ for $P$. fluorescens and $\geq 133 \mathrm{~J} \mathrm{~cm}^{-2}$ for S. epidermidis, with $400 \mathrm{~nm}$ peaking light (violet). However, P. fluorescens required only $4 \mathrm{~J} \mathrm{~cm}^{-2}$ when blue light was used. Figure 4 also displays the specific bacterial trends. Regarding P. fluorescens inactivation (Figure 4, round legend symbols), a little increase in dose for $1-\log _{10}$ reduction was required as irradiance increased. However, an opposite trend was evident in S. epidermidis data (Figure 4, squared legend symbols), where a higher irradiance resulted in a decrease of the dose for $1-\log _{10}$ reduction. 


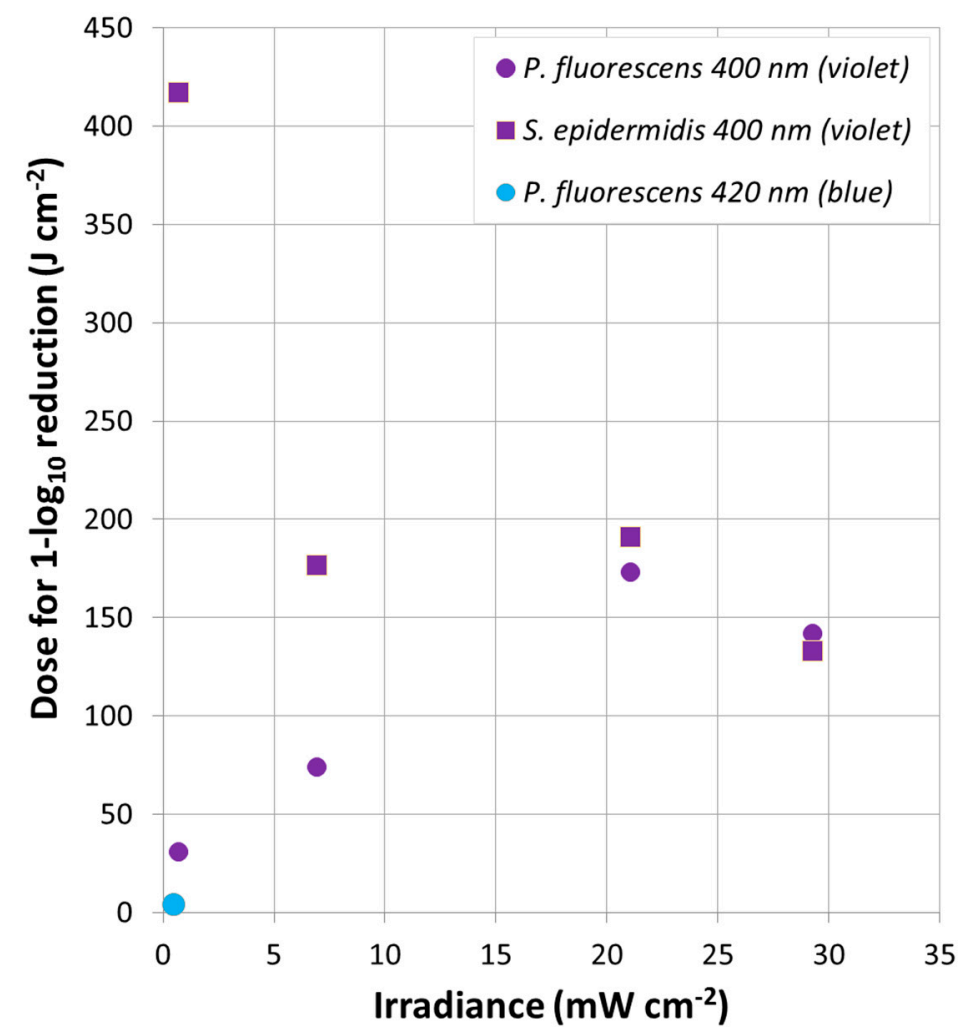

Figure 4. Dose for 1-log reduction. Dose for $1-\log _{10}$ reduction as a function of the irradiance. P. fluorescens (round symbols) and S. epidermidis (squared symbols) are represented in violet color for $400 \mathrm{~nm}$. The treatment with $100 \%$ of $\mathrm{I}_{\max }$ using $420 \mathrm{~nm}$ (blue light) is represented in blue (only for P. fluorescens).

\section{Discussion}

This study investigated the antibacterial effect of a high-tech optical set up, composed of an exchangeable LED array emitting in the visible range, on P. fluorescens and S. epidermidis biofilms. The impact of several LED arrays, characterized by different wavelengths and irradiances, was examined. In this research, biofilms were grown on a plastic (polystyrene) surface to facilitate the extension of this study to real contaminated surfaces within the food, healthcare, households and hospital settings [58]. Future studies should account for different (bio)materials and assess the photodynamic inactivation impact on non-flat surfaces as well.

\subsection{P. fluorescens and S. epidermidis Inactivation by LEDs}

The most evident outcome, resulting from the experiments carried out, was the inactivation of the Gram-negative P. fluorescens and Gram-positive S. epidermidis biofilms, utilizing violet LED light. In contrast, the performed experiments proved neither promotion nor reduction of biofilm inactivation when green, yellow and red light exposure was employed. Blue light had an exceptional impact depending on the bacterial biofilms which were tested: it had a killing effect on P. fluorescens (with $100 \%$ of $\mathrm{I}_{\max }$ ) while it was harmless for S. epidermidis.

\subsubsection{Violet Light}

A previous study, conducted with violet light (405 nm LED) on planktonic bacteria, found a reduction of $1.0 \log _{10}$ within the population [52]. The bacteria undergoing the treatment were the food pathogen Staphylococcus aureus, while refrigerated $\left(4{ }^{\circ} \mathrm{C}\right)$, for a treatment time of $7.5 \mathrm{~h}$ and a total dose of $486 \mathrm{~J} \mathrm{~cm}^{-2}$. The same authors attributed the antibacterial effect induced by the LED light to physical damage of the bacterial wall rather than to DNA destruction: DNA was found not to be damaged [52]. Ferrer-Espada et al. who focused their work on biofilms grown using a CDC reactor, found a reduction 
of 1.88, 2.78 and $3.18 \log _{10}$ for Acinetobacter baumannii, Escherichia coli and Pseudomonas aeruginosa irradiated with $405 \mathrm{~nm}$ [32]. They exposed biofilms to doses of 162, 576 and $500 \mathrm{~J} \mathrm{~cm}^{-2}$ respectively, compared to $420 \mathrm{~J} \mathrm{~cm}^{-2}$ used in the current research. Inactivation was claimed by Halstead et al. as well, who investigated $400 \mathrm{~nm}$ light against 34 isolates and found violet light effective against P. aeruginosa and S. aureus [53]. Based on these findings and the literature, there is no doubt about the antibacterial activity of the violet light [32,40,41,43,49,51,54,56,63-67]. However, the evaluation of its effectiveness and a direct comparison of the results, obtained by different studies, are more complicated. The complexity is due to the usage of planktonic suspensions rather than biofilms, different protocols used to grow biofilms, different initial populations, models used for the inactivation, exposure times, supplied doses, irradiances and, finally, specific bacterial properties and features (PSs contents and types) under investigation. In this work, the possibility to change the irradiance emitted by the set up allowed for the investigation of several irradiances. It was found that the stronger the violet irradiance became, the lower the residual population, $N_{\text {res }}$, was. This was valid for both examined bacteria and it is in complete agreement with the literature focused on the UV range, where powerful sources promoted complete eradication [23]. The findings of Section 2.5 also demonstrated that inactivation (light-killed population) of P. fluorescens biofilms was stronger than S. epidermidis biofilms when the total dose irradiating the samples was lower (2.5\% and 25\% $\mathrm{I}_{\max }$, Figure 3 in Section 2.5$)$. It can be conceivably hypothesized that the difference between $P$. fluorescens and S. epidermidis inactivation may be due to the presence of different endogenous PSs within each bacterial species having different absorption spectra [38-41,47]. Another reason may be the different quantity of PSs absorbing light per bacterial cell: a higher concentration leads to increased reactive oxygen species production and, therefore, a stronger killing effect under lower irradiation conditions. The level of endogenous PSs is indeed an intrinsic characteristic of the strain. Moreover, the probability of photoexcitation of a PS is driven by the direct interaction, photons-PS. Because the two species are characterized by a different cell wall as a result of the Gram-nature, the physiological and morphological structure of the bacteria played a role as well. This aspect is discussed in Section 3.3.

\subsubsection{Blue Light}

In some works, blue light (420-470 nm) has been tested as well, showing a strong effect against both Gram-positive (Bacillus cereus, Listeria monocytogenes and methicillin-resistant S. aureus) and Gram-negative pathogens (P. aeruginosa, Salmonella Typhimurium and E. coli) in their planktonic form [39-41,49,50,54,56,65-68]. However, Abana et al. claimed that the effect of $455 \mathrm{~nm}$ is not completely bactericidal on E. coli but depends on the microorganism (pathogenic or non-pathogenic) and growth phase. They demonstrated that the stationary phase was more resistant compared to the exponential phase [45]. Based on our findings, blue light only had an impact on P. fluorescens biofilms under the harshest condition $\left(100 \% \mathrm{I}_{\max }\right)$. No evidence of inactivation was observed on S. epidermidis biofilms. The inactivation obtained with the blue light on $P$. fluorescens biofilms correlates favorably with Amin et al. and further supports the idea that $P$. fluorescens likely contains porphyrins absorbing specific wavelengths of the blue emission spectrum (420 nm for instance) that might promote cell death [37]. Hyun and Lee observed that P. fluorescens compared to L. monocytogenes was found to be the most sensitive strain to blue light treatment [50]. However, these authors were using a blue light peaking at $460 \mathrm{~nm}$ and investigating the effect on bacteria inoculated and left dry on packaging materials (no biofilms). The light provoked disruption of the cell membrane, and release of intracellular component post-treatment [50].

\subsubsection{Green, Yellow and Red Light}

The poor performances (unaltered population density) obtained with green, yellow and red light exposure in terms of inactivation were in contrast with some works that, instead, evidenced the influence of this visible range on bacteria. Some studies performed on planktonic form showed reduction or promotion on bacterial cells depending on the temperature at which the experiments 
were performed. Ghate et al. investigated the impact of 521 and $642 \mathrm{~nm}$ on four different pathogens at different temperatures $\left(20,15\right.$ and $\left.10^{\circ} \mathrm{C}\right)$ for a total exposure time of $7.5 \mathrm{~h} \mathrm{[49]}$. At $20{ }^{\circ} \mathrm{C}$, they found an increase of the population densities (approximately $1.5 \log _{10}$ ) when the four bacteria were exposed to one of these two wavelengths. At 15 and $10^{\circ} \mathrm{C}$, instead, a reduction was observed when bacteria were irradiated at $521 \mathrm{~nm}$. Specifically, 1.7, 1.7, 1.0 and $0.9 \log _{10}$ at $15{ }^{\circ} \mathrm{C}$ and $1.8,1.7,1.5$ and $1.5 \log _{10}$ $10{ }^{\circ} \mathrm{C}$ for S. Typhimurium, S. aureus, E.coli and L. monocytogenes, respectively. No variation within the population densities was found at $642 \mathrm{~nm}$.

Other studies showed that the bacterial species under investigation played a crucial role on the inactivation. In fact, Kim et al. found a reduction up to $60 \%$ and $40 \%$ on S. aureus and E. coli, respectively [48]. On the contrary, they found an increase of the population of 2.5-fold after $8 \mathrm{~h}$ for Pseudomonas gingivalis. These experiments were performed with irradiation at $525 \mathrm{~nm}$ on planktonic cells. Yu and Lee, on one hand, found that green light promoted the antifungal activity of Bacillus amyloliquefaciens but, on the other hand, claimed that it reduced mycelial growth on Colletotrichum acutatum $[57,58]$.

Then, there are other studies where a reduction is observed regardless of the two above-mentioned temperature and species. Ghate et al. studied the effect of 461 and $521 \mathrm{~nm}$ against the pathogens E. coli, S. Typhimurium and L. monocytogenes [46]. Treatments of $7.5 \mathrm{~h}$ were applied with a total dose ranging from 100 to $600 \mathrm{~J} \mathrm{~cm}^{-2}$ and led to the conclusion that $521 \mathrm{~nm}$ decreased the bacteria in their planktonic form. The efficiency of the green light treatment was lower than blue light but still, it caused a reduction of $1-2 \log _{10} \mathrm{CFU} \mathrm{mL} \mathrm{mL}^{-1}$ at both acidic and alkaline conditions. Kumar et al. also demonstrated at $520 \mathrm{~nm}$ a reduction of $1.2 \log _{10}$ at $25^{\circ} \mathrm{C}$ of $S$. aureus and $0.7 \log _{10}$ at 25,10 and $4{ }^{\circ} \mathrm{C}$ of L. monocytogenes. Some studies performed on fungi showed similar impacts [30].

Brito Aragão et al. focused, instead, on biofilms. They tested two different red peaking LEDs with an emission range of 620-660 nm and found that irradiation alone was causing no reduction within the population [55], while Yu and Lee found enhanced biofilms' formation and motility on B. amyloliquefaciens [57]. Even though these wavelengths were chosen because they showed growth enhancing or bactericidal effects, none of these effects was observed against biofilms of $P$. fluorescens and S. epidermidis under different conditions. This research demonstrated the ineffectiveness of these wavelengths in terms of inactivation of two specific bacterial biofilms. Future research should be focused on the use of the examined ineffective wavelengths to promote antibacterial activity in synergy with exogenous PSs $[27,44,69,70]$.

\subsection{Sublethal Injury}

Although the sublethal injury (SI) kinetics have completely different trends and percentages of injury, confirming the response to the treatments depends on the microorganism, a generalization on the light impact is possible. The SI curves for violet LEDs demonstrate that for both microorganisms, the percentages of SI increase when the irradiance increases. This can be easily explained: the number of photons reaching the biofilms is higher and consequently, so is the sublethal injury. However, the tendency of the SI is slightly different compared to what was found in other research. Ghate et al. determined that the SI only increased with the exposure time [46]. In contrast, in this study, it reaches a plateau or a peak. This is probably due to the difference in population growth forms: planktonic compared to biofilms.

\subsection{Factors Influencing the Inactivation Kinetics}

Several factors influenced the kinetics of the photoinactivation: (i) the Gram-nature and the intrinsic difference within the developed biofilms belonging to the two species, (ii) the endogenous PSs within microorganisms, (iii) the direct impact on enzymes and (iv) the production of specific molecules by microorganisms.

First of all, regarding the Gram-nature, an important result that emerged from this study is that Gram-negative P. fluorescens and Gram-positive S. epidermidis biofilms, responded with the same 
photoinactivation to a treatment with violet light at the two highest irradiances $(100 \%$ and $75 \%$ of $I_{\max }$ ): for both species, populations were inactivated with $3 \log _{10}$ and $1.5 \log _{10}$ without any significant differences between the two microorganisms (Section 2.5). These findings are in contradiction with previous studies reported in the literature, where some authors confirmed a stronger inactivation effect on Gram-positive microorganisms, which have a different cell wall lacking both outer membrane and lipopolysaccharide layer but have a thicker peptidoglycan layer [51]. They are also in contrast with others claiming that the most sensible bacteria are the Gram-negative ones, even though they are characterized by a more complex cell wall [53]. Halstead et al. found that among 34 isolates, the most susceptible were the Gram-negative bacteria A. baumannii ACI_19606, for which a 93.5\% reduction was observed after $15 \mathrm{~min}$ of light exposure [53]. The intrinsic differences between the two types of bacteria (Gram-nature) and the biofilm they formed is also an interesting aspect. A comparison between the curves of the controls for P. fluorescens and S. epidermidis showed how the biofilms, developed using the two strains, reacted differently under drying conditions. P. fluorescens underwent a process of desiccation that can justify the reduction in the biofilm population (no light-exposure), while the S. epidermidis biofilms did not show any reduction. Gram-negative bacteria seemed more sensitive to drying than Gram-positive, as previous authors confirmed [15]. The comparison between the kinetics of $P$. fluorescens and S. epidermidis requires further comments. Although the inactivation kinetics are alike in shape, they are slightly different regarding the time frame of the log-linear phase, which was shorter for S. epidermidis compared to P. fluorescens.

Then, based on the literature that focused on the investigation of the endogenous PSs responsible for the photoinactivation and considering the absorption spectra of the porphyrins and flavins, it is possible to claim that the wavelengths with the strongest antibacterial effect are the ones belonging to the main absorption peak: violet-blue range [38-41,47]. Although the other wavelengths of the visible spectra followed in correspondence with the secondary absorption peaks of the same chromophores, they were harmless [38-41,47].

Another factor that can be involved is the direct damage caused by photons to the enzymes succinate dehydrogenase (SDH) and lactate dehydrogenase (LDH). This process does not involve reactive oxygen species production [71]. The damage mechanism is due to the tail of the violet LED spectrum continuing in the UV-A spectral region, which is responsible for their destruction [71]. Finally, the last factor that may play a role in the detrimental effect of light on P. fluorescens biofilms is its effect on the production of pyocyanin or pyoverdine (siderophores), blue extracellular products that may contribute to light absorption $[67,72]$.

\subsection{Doses and Bacterial Trends}

The analyses of the dose required for $1 \log _{10}$ reduction highlighted the impact of violet or blue exposure on P. fluorescens and S. epidermidis biofilms as antibacterial treatment, compared to the other ineffective wavelengths (blue, green, yellow and red). Concerning P. fluorescens, even better inactivation was achieved with blue light rather than violet (Figure 4). A remarkable difference in the dose required for $1 \log _{10}$ reduction between blue and violet light was observed. The blue dose required to reduce the bacteria population in the biofilm was found to be 8 times higher compared to the violet dose (Section 2.5). A lower dose means lower energy requirement per area and translates into lower energy consumption and lower costs, thus representing an advantage as new technology.

The examination of the bacterial trends (dose for $1 \log _{10}$ reduction as a function of irradiance) demonstrated interesting features. It was found that the dose for $1 \log _{10}$ reduction increases asymptotically as the irradiance increases for P. fluorescens biofilms, whereas it decreases asymptotically as the irradiance increases for S. epidermidis biofilms. Tomb et al. observed the opposite trend for P. fluorescens (required dose decreases as a function of irradiance), although S. epidermidis' trend is in line and practically the same as the one proposed by the same authors [67]. It was hypothesized that the opposite trend might be due to a saturation effect. The excess of irradiance does not enhance the 
number of excited endogenous PSs within P. fluorescens cells that might be previously promoted to an excited molecular state [73].

The presence of a tail in the kinetics, obtained using the Geeraerd et al. model, made clear that the inactivation with violet light after $180 \mathrm{~min}$ can no more and no longer increase the number of dead cells [62]. An increase in the exposure time after a certain cut off value is useless and does not promote any additional activation. This is an important aspect that is first different in planktonic form where cells are free to float [46], compared to the situation where the cells are trapped within the matrix (biofilm). Moreover, the exposure time in this work is an average between the long treatments, such as the 4 days of exposure tested by Hyun et al. against P. fluorescens and L. monocytogenes [50] and the short exposures, like Rupel et al. who used treatment times of a few minutes. The latter used a powerful laser source at $455 \mathrm{~nm}$, assuring an effect on a short time but using a light source characterized by high energy consumption and cost compared to LEDs [74].

\section{Materials and Methods}

\subsection{Bacterial Strains and Pre-Culture Preparation}

A single colony of the Gram-negative Pseudomonas fluorescens (ATCC ${ }^{\circledR} 13,525$ culti-loops, Thermo Fisher Scientific, Waltham, MA, USA) or Gram-positive Staphylococcus epidermidis (NCTC 11,047 Lenticule discs, St. Luis, MI, USA) was placed in $20 \mathrm{~mL}$ broth. Tryptic Soy Broth (TSB, VWR Chemicals, Radnor, PA, USA) and Luria Bertani (LB, Becton Dickinson, Franklin Lakes, NJ, USA) supplemented with $5 \mathrm{~g} \mathrm{~L}^{-1} \mathrm{NaCl}$, were used for P. fluorescens and S. epidermidis, respectively. The pre-cultures were grown at the constant temperature of $25^{\circ} \mathrm{C}$ (P. fluorescens) and $37^{\circ} \mathrm{C}$ (S. epidermidis) for $24 \mathrm{~h}$, with shaking (160 rpm).

\subsection{Biofilm Growth}

Bacterial biofilms were grown on the polystyrene surface of petri dishes (50 mm diameter, $8 \mathrm{~mm}$ height, Simport, Canada). $400 \mu \mathrm{L}$ of inoculum suspension, of approximately $10^{7} \mathrm{CFU} \mathrm{mL}^{-1}$, was spread on a circular area $\left(7.0 \mathrm{~cm}^{2}\right)$ with a plastic loop. After inoculation, the petri dishes were closed and left to grow for $24 \mathrm{~h}$ at a constant temperature of $25^{\circ} \mathrm{C}$ (P. fluorescens) and $20^{\circ} \mathrm{C}$ (S. epidermidis), under sterile conditions.

\subsection{Rinsing Procedure}

After $24 \mathrm{~h}$, the mature biofilms had grown at the solid-liquid interface. The excess of bacterial suspension was removed using a pipette. $3 \mathrm{~mL}$ of Phosphate Buffer Saline (PBS, Sigma Aldrich, Saint Louis, MO, USA) solution was poured into the petri dish and then gently shaken. Finally, the PBS was carefully removed. The rinsing procedure was repeated twice. The biofilm was then ready to be treated using light or for quantification.

\subsection{Light Set up and Optical Conditions}

An array composed of 120 LEDs was used as the light source for the investigation. The violet LEDs (UVC5TZ-400-30) were purchased from Bivar (California) while the blue (L-53MBD), green (L-1503CGDK), yellow (L-53YD) and red (L-53HD) were purchased from Kingbright (Taiwan). All of them had an emission in the visible region of the light spectrum. They were optically characterized in terms of wavelength, irradiance and emission in time. The setup was provided with a mechanism that allowed for the replacement of the LED array. A liquid crystal display allowed the operator to change the electric current flowing through the LEDs (0-30 mA), and thus, the power density emitted by the light source. Different conditions were investigated for each color: $100 \%, 75 \%$ and $25 \%$ of the maximum irradiances $\left(\mathrm{I}_{\max }\right)$ were tested on the bacterial biofilms. For the violet LED array, $2.5 \%$ was explored as well, to obtain an irradiance comparable to the other LED sources. The violet-emitted irradiance was measured using a radiometer RM-12 and sensor (UVA+ 339-440 nm) from Opsytec Dr. 
Grobel (Germany). The spectrometer MF250N from UPRtek Corp. (Taiwan) was used for the other LED colors. The total dose for each condition was calculated as follows [51]:

$$
\text { Total dose }=\text { Irradiance } \times t_{\mathrm{TOT}}
$$

where $\mathrm{t}_{\mathrm{TOT}}$ represents the total exposure time equal to $4 \mathrm{~h}$. It represents the total energy delivered for the irradiated area. Figure 5 displays a scheme of the set up (Figure 5a), two pictures of the set up (top, Figure $5 c$, and gross section, Figure $5 d$ ) and the LEDs matrices (Figure 5b) used during the experiment.

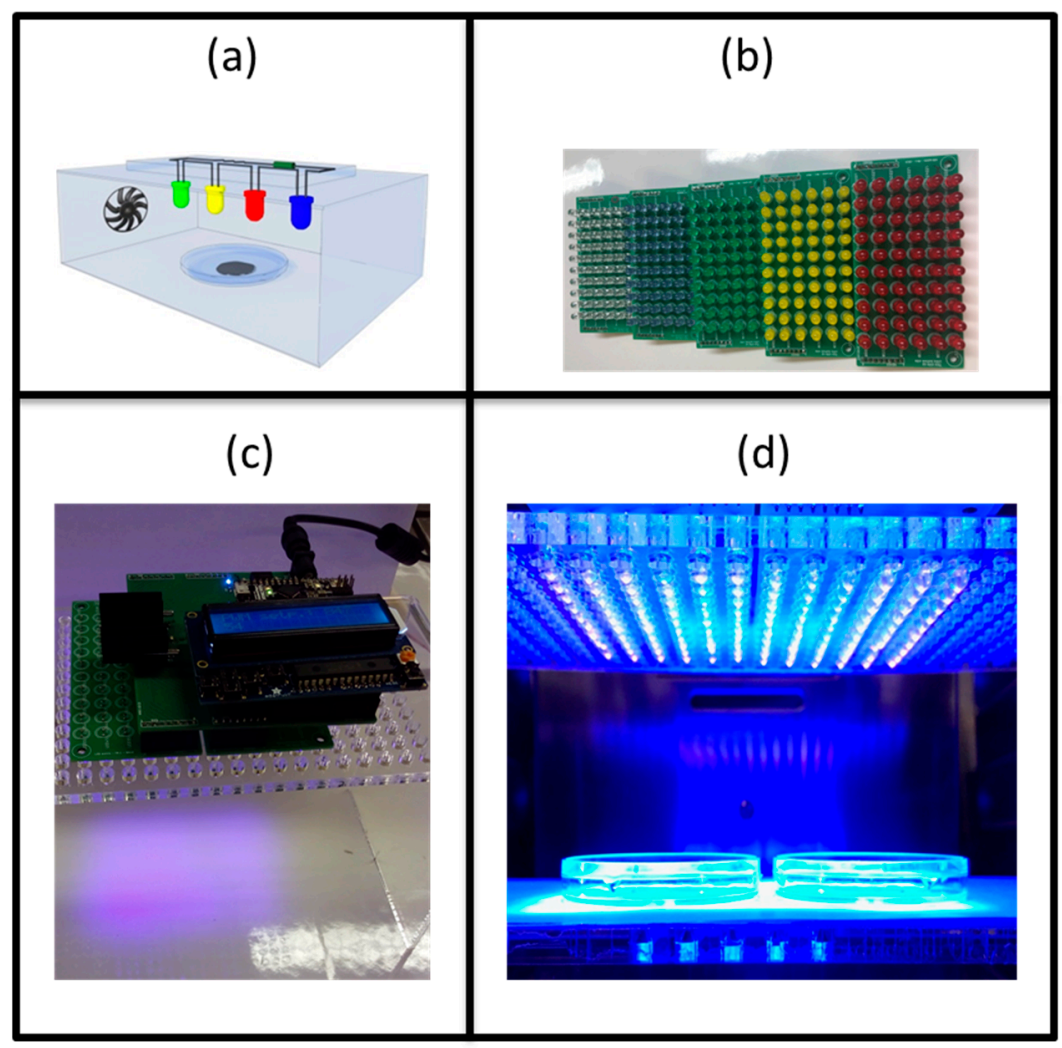

Figure 5. Optical set up: (a) scheme of the set up inside the incubator, (b) mountable matrices used in the experiments, from right to left the light colors are violet, blue, green, yellow and red, (c) set up with liquid-crystal display on the top and (d) cross-section of the set up in which the LED matrices are irradiating the biofilm in the petri dishes.

\subsection{Biofilm Treatments}

Mature biofilms were treated for different light exposure times: 30, 60, 90, 120, 150, 210 and $240 \mathrm{~min}$. The distance array-samples equaled $6.5 \mathrm{~cm}$ and the temperature during the whole experiment was kept constant at $20^{\circ} \mathrm{C}$ by placing the set up inside an incubator. The biofilms were placed open underneath the LED array. The control referred to biofilms in the dark that did not undergo the light treatment. The control biofilms were placed (open) inside the incubator and could be dried by the air.

\subsection{Biofilm Quantification}

After the treatment, $2 \mathrm{~mL}$ of PBS solution was added to each biofilm. The biofilm was detached from the surface using a cell scraper (20 mm blade, Carl Roth, Karlsruhe, Germany). Afterwards, the suspension was homogenized with a pipette, it became overall turbid and no pellicles were floating in it. Serial dilution suspensions were prepared in saline solution $(0.85 \% \mathrm{NaCl})$ and these suspensions were plated both on general medium (GM) and on selective medium (SM). Tryptic Soy Agar (TSA) and CFC-supplemented-Pseudomonas Agar Base (Cetrimide-Fucidin-Cephalosporin-PAB, Thermo 
Scientific, Waltham, Massachusetts, United States) were used as GM and SM for P. fluorescens. While for S. epidermidis, brain heart infusion (BHI) supplemented with $13 \mathrm{~g} \mathrm{~L}^{-1}$ of bacteriological agar (Merck, Darmstadt, Germany) and Staphylococcus agar containing $75 \mathrm{~g} \mathrm{~L}^{-1} \mathrm{NaCl}$ were used as GM and SM, respectively.

\subsection{Inactivation Model}

The Geeraerd et al. inactivation model was used to fit the light-response data [62]. This non-linear model is based on a sigmoidal-like curve with a shoulder (lag phase), a log-linear inactivation and a tail (indicating the existence of a residual population surviving the inactivation treatment). The data collected in this work did not show any evidence of a shoulder, so the model was simplified to the model without shoulder expressed by the following (static) equation:

$$
N(t)=\left(N_{0}-N_{\text {res }}\right) \times \exp \left(-k_{\max } \times t\right)+N_{\text {res }}
$$

or in some cases, to the model without shoulder and without tail, which was reduced to the classical log-linear regression model:

$$
N(t)=N_{0} \times \exp \left(-k_{\max } \times t\right) .
$$

In these equations, $N(t)\left(\mathrm{CFU} \mathrm{\textrm {cm } ^ { - 2 }}\right)$ represents the biofilm microbial population at time $t$ (min), $N_{0}\left(\mathrm{CFU} \mathrm{cm}{ }^{-2}\right)$ represents the initial cell density, $k_{\max }\left(\mathrm{min}^{-1}\right)$ is the maximum inactivation rate and $N_{\text {res }}\left(\mathrm{CFU} \mathrm{cm}{ }^{-2}\right)$ is the biofilm residual population. The difference between the initial population $\left(N_{0}\right)$ and the residual population $\left(N_{\text {res }}\right)$ is referred to as $\log _{10}$ reduction. During parameters estimation, logarithmically transformed data are used. The root mean squared error (RMSE) between the observed $\left(N_{\text {exp }}\right)$ and predicted $\left(N_{\text {mod }}\right) \log _{10}$ values was used to evaluate the goodness of the fit,

$$
\text { RMSE }=\sqrt{\frac{\sum_{1}^{n}\left(N_{\text {exp }}(t i)-N_{\text {mod }}(t i)\right)^{2}}{n-p}},
$$

where $n$ is the number of measurements and $p$ is the number of estimated parameters.

\subsection{Sublethal Injury}

The sublethal injury (SI), consequent to light exposure, was calculated using the following equation [75]:

$$
\text { Sublethal Injury }(\%)=\frac{\text { Cell Counts on GM }- \text { Cell Counts on SM }}{\text { Cell Counts on GM }} \times 100 \% \text {. }
$$

SI is calculated based on the continuous fit of the Geeraerd et al. model [62]. It gives a percentage related to the damage caused by light exposure.

\subsection{Dose for $1 \log _{10}$ Reduction}

The estimation of the parameter $N_{\text {res }}$ of the control (no light exposure) and of the light-exposed biofilms allowed for the calculation of the light dose $\left(\mathrm{J} \mathrm{cm}^{-2}\right)$ required to reduce the bacterial population with $1 \log _{10}(90 \%)$. The required dose gave an idea of the strength of the killing potential of the light treatment. The dose for $1 \log _{10}$ reduction has been calculated as:

$$
\text { Dose for } 1 \log _{10} \text { red }=\frac{\text { Total dose }}{N_{\text {res }} \text { control }-N_{\text {res }} \text { light }}
$$

where $N_{\text {res }}$ was estimated by the fitting of the Geeraerd et al. model [62]. 


\subsection{Statistical Analysis}

To statistically compare the estimated parameters evaluated using the Geeraerd et al. model, a one-way analysis of variance (ANOVA) was carried out using a multiple comparison tests based on the multcompare function implemented in MATLAB R2016a (The Math Works, Inc.) [62]. A confidence level of $95 \%$ was used to assess the significant difference. A $p$-value less than 0.05 was considered significantly different.

\section{Conclusions}

The number of photodynamic inactivation studies has greatly increased in recent years. These studies have mainly examined planktonic cells [38-41,43,45-52], while few studies have taken bacterial biofilms into consideration [16,30,32,50-55]. Since biofilms pose a major global healthcare problem, the impact of photodynamic inactivation on them is both crucial and innovative.

Previous research on the antimicrobial potential of visible light has focused mainly on bacteria growing in a planktonic form and to a lesser extent, on biofilms, which have been identified as a crucial problem for hygiene control in households, industries and clinical environments in the last years. Therefore, this research focused on the effect of five different wavelengths of visible LED light on the inactivation dynamics of mature biofilms. Moreover, this research included both a Gram-negative and Gram-positive bacterial strain, as the Gram-nature is seen as an important factor in the resistance against antimicrobial treatments. This work adds to a growing body of research on the effect of photodynamic inactivation against biofilms. The broad study explores the effect of several wavelengths and irradiance levels on common biofilm-formers with a different Gram-nature. As such, it provides insights into (the limits of) the potential of LED light as an antibiofilm strategy.

The results demonstrated that the high-energy violet light was able to inactivate biofilms of both the Gram-negative P. fluorescens and Gram-positive S. epidermidis. Blue light also had an inactivating effect on P. fluorescens but not on S. epidermidis. This demonstrates the higher resistance of the Gram-positive biofilms. Green, yellow and red light had no effect on the biofilm population compared to untreated control samples. Even though biofilms are more antimicrobial-resistant than planktonic bacteria, this research demonstrated the potential of simple visible light as an anti-biofilm strategy. However, the research also demonstrated that this potential is limited to violet light when considering a treatment that should be effective against a broad spectrum of bacteria.

Bacterial inactivation by photons is hypothesized to be mediated by reactive oxygen species. Future work should focus on improving the understanding of the inactivation mechanism of visible light. Such an improved understanding will allow further finetuning of this promising technology.

Author Contributions: Individual contributions of each of the authors to the research are the following: Conceptualization, V.A., C.S., S.A., A.C. and J.F.M.V.I.; methodology, V.A.; software, V.A.; validation, V.A.; formal analysis, V.A. and C.W.; investigation, V.A. and C.W.; resources, V.A.; data curation, V.A.; writing-original draft preparation, V.A.; writing-review and editing, V.A., C.S., S.A. and J.F.M.V.I.; visualization, V.A.; supervision, C.S., A.C. and J.F.M.V.I.; project administration, V.A.; funding acquisition, J.F.M.V.I. All authors have read and agreed to the published version of the manuscript.

Funding: This research was funded by project Bioclean Marie Skłodowska-Curie Actions-Innovative Training Networks (MSCA-ITN) 722871 of the HORIZON 2020 EU Framework Programme for Research and Innovation, by project C24/18/046 of the KU Leuven Research Council and by project G.0863.18 of the Research Foundation-Flanders. S.A. was supported by the Research Foundation-Flanders, under grant 1224620N.

Acknowledgments: The authors thank Bart Huyck for his contribution to design and build the optical set up used in the current research.

Conflicts of Interest: The authors declare no conflict of interest.

\section{References}

1. Wang, B.; Tan, X.; Du, R.; Zhao, F.; Zhang, L.; Han, Y.; Zhou, Z. Bacterial composition of biofilms formed on dairy-processing equipment. Prep. Biochem. Biotechnol. 2019, 50, 477-484. [CrossRef] [PubMed] 
2. Flemming, H.; Wingender, J. The biofilm matrix. Nat. Rev. Microbiol. 2010, 8, 623-633. [CrossRef]

3. Leriche, V.; Sibille, P.; Carpentier, B. Use of an enzyme-linked lectinsorbent assay to monitor the shift in polysaccharide composition in bacterial biofilms. Appl. Environ. Microbiol. 2000, 66, 1851-1856. [CrossRef] [PubMed]

4. Mann, E.E.; Wozniak, D.J. Pseudomonas biofilm matrix composition and niche biology. Fems Microb. Rev. 2012, 36, 893-916. [CrossRef] [PubMed]

5. Seviour, T.; Derlon, N.; Du, M.; Flemming, H.-C.; Girbal-Neuhauser, E.; Horn, H.; Kjelleberg, S.; van Loosdrecht, M.C.M.; Lotti, T.; Malpei, M.F.; et al. Extracellular polymeric substances of biofilms: Suffering from an identity crisis. Water Res. 2019, 151, 1-7. [CrossRef] [PubMed]

6. Zhang, T.; Fang, H.H. Quantification of extracellular polymeric substances in biofilms by confocal laser scanning microscopy. Biotechnol. Lett. 2001, 23, 405-409. [CrossRef]

7. Cochran, W.L.; McFeters, G.A.; Stewart, P.S. Reduced susceptibility of thin Pseudomonas aeruginosa biofilms to hydrogen peroxide and monochloramine. J. Appl. Microbiol. 2000, 88, 22-30. [CrossRef]

8. Luque-Sastre, L.; Fox, E.M.; Jordan, K.; Fanning, S. A comparative study of the susceptibility of Listeria species to sanitizer treatments when grown under planktonic and biofilm conditions. J. Food Prot. 2018, 81, 1481-1490. [CrossRef]

9. Majumdar, S.; Pal, S. Cross-species communication in bacterial world. J. Cell Commun. Signal. 2017, 11, 187-190. [CrossRef]

10. Mah, T.F.; O'Toole, G.A. Mechanisms of biofilm resistance to antimicrobial agents. Trends Microbiol. 2001, 9, 34-39. [CrossRef]

11. Bryers, J.D. Medical biofilms. Biotechnol. Bioeng. 2008, 100, 1. [CrossRef] [PubMed]

12. Muhammad, J.; Khan, S.; Su, J.Q.; El-Latif Hesham, A.; Ditta, A.; Nawab, J.; Ali, A. Antibiotics in poultry manure and their associated health issues: A systematic review. J. Soils Sediments 2020, 20, 486-497. [CrossRef]

13. Vestby, L.; Møretrø, T.; Langsrud, S.; Heir, E.; Nesse, L. Biofilm forming abilities of Salmonella are correlated with persistence in fish meal- and feed factories. Bmc Vet. Res. 2009, 5, 20. [CrossRef] [PubMed]

14. Iñiguez-Moreno, M.; Gutiérrez-Lomel, M.; Avila-Novoa, M.G. Kinetics of biofilm formation by pathogenic and spoilage microorganisms under conditions that mimic the poultry, meat, and egg processing industries. Int. J. Food Microbiol. 2019, 303, 32-41. [CrossRef]

15. Røssvoll, E.; Langsrud, S.; Bloomfield, S.; Moen, B.; Heir, E.; Møretrø, T. The effects of different hygiene procedures in reducing bacterial contamination in a model domestic kitchen. J. Appl. Microbiol. 2015, 119, 582-593. [CrossRef]

16. Song, H.H.; Lee, J.K.; Um, H.S.; Chang, B.S.; Lee, S.Y.; Lee, M.K. Phototoxic effect of blue light on the planktonic and biofilm state of anaerobic periodontal pathogens. J. Periodontal. Implant. Sci. 2013, 43, 72-78. [CrossRef]

17. Marti, R.; Schmid, M.; Kulli, S.; Schneeberger, K.; Naskova, J.; Knøchel, S.; Ahrens, C.H.; Hummerjohann, J. Biofilm formation potential of heat-resistant Escherichia coli dairy isolates and the complete genome of multidrug-resistant, heat-resistant strain FAM21845. Appl. Environ. Microbiol. 2017, 83, 15. [CrossRef]

18. Kip, N.; van Veen, J. The dual role of microbes in corrosion. ISME J. 2015, 9, 542-551. [CrossRef]

19. Govaert, M.; Smet, C.; Baka, M.; Ećimović, B.; Walsh, J.L.; Van Impe, J. Resistance of L. monocytogenes and S. Typhimurium towards Cold Atmospheric Plasma as function of biofilm age. Appl. Sci. 2018, 8, 2702. [CrossRef]

20. Rai, M.; Ingle, A.; Gaikwad, S.; Gupta, I.; Gade, A.; Silvério da Silva, S. Nanotechnology based anti-infectives to fight microbial intrusions. J. Appl Microbiol. 2005, 33, 78-82. [CrossRef]

21. Carmen, J.; Roeder, B.; Nelson, J.; Ogilvie, R.; Robison, R.; Schaalje, G.B.; Pitt, W.G. Treatment of biofilm infections on implants with low-frequency ultrasound and antibiotics. Am. J. Infect. Control. 2005, 120, 3, 527-542. [CrossRef]

22. Stiefel, P.; Mauerhofer, S.; Schneider, J.; Maniura-Weber, K.; Rosenberg, U.; Ren, Q. Enzymes enhance biofilm removal efficiency of cleaners. Antimicrob. Agents Chemother. 2016, 60, 3647-3652. [CrossRef]

23. Kim, D.; Kang, D. Elevated inactivation efficacy of a pulsed UVC Light-Emitting Diode system for foodborne pathogens on selective media and food surfaces. Appl. Environ. Microbiol. 2018, 84, 20. [CrossRef]

24. Lipovsky, A.A.; Nitzan, Y.; Lubart, R. A possible mechanism for visible light-induced wound healing. Lasers Surg. Med. 2008, 40, 509-514. [CrossRef] 
25. Tavares, A.; Carvalho, C.M.B.; Faustino, M.A.; Neves, M.G.P.M.S.; Tomé, J.P.C.; Tomé, A.C.; Cavaleiro, J.A.S.; Cunha, Â.; Gomes, N.C.M.; Alves, E.; et al. Antimicrobial photodynamic therapy: Study of bacterial recovery viability and potential development of resistance after treatment. Mar. Drugs 2010, 8, 91-105. [CrossRef]

26. Maresca, J.A.; Keffer, J.L.; Hempel, P.P.; Polson, S.W.; Shevchenko, O.; Bhavsar, J.; Powell, D.; Miller, K.J.; Singh, A.; Hahn, M.W. Light modulates the physiology of nonphototrophic Actinobact. J. Bacteriol. 2019, 24, 201. [CrossRef]

27. De Souza, S.; Junqueira, J.; Balducci, I.; Koga-Ito, C. Photosensitization of different Candida species by low power laser light. J. Photochem. Photobiol. B Biol. 2006, 83, 34-38. [CrossRef]

28. Oms-Oliu, G.; Martín-Belloso, O.; Soliva-Fortuny, R. Pulsed light treatments for food preservation. A Review. Food Bioprocess. Technol. 2010, 3, 13. [CrossRef]

29. Feuerstein, O. Light therapy: Complementary antibacterial treatment of oral biofilm. Adv. Dent. Res. 2012, 24, 103-107. [CrossRef]

30. Kumar, A.; Ghate, V.; Kim, M.-J.; Zhou, W.; Khoo, G.H.; Yuk, H.-G. Kinetics of bacterial inactivation by $405 \mathrm{~nm}$ and $520 \mathrm{~nm}$ light emitting. J. Photochem. Photobiol. B Biol. 2015, 149, 37-44. [CrossRef]

31. Lukšiene, Ž. New approach to inactivation of harmful and pathogenic microorganisms by photosensitization. Food Technol. Biotechnol. 2005, 43, 411-418. [CrossRef]

32. Ferrer-Espada, R.; Liu, X.; Goh, X.S.; Sharo, X. Antimicrobial blue light inactivation of polymicrobial biofilms. Front. Microbiol. 2019, 10, 721. [CrossRef]

33. Nitzan, Y.; Kauffman, M. Endogenous porphyrin production in bacteria by $\delta$-aminolaevulinic acid and subsequent bacterial photoeradication. Lasers Med. Sci. 1999, 14, 269. [CrossRef]

34. Soukos, N.S.; Som, S.; Aberne, A.D.; Ruggiero, K.; Dunham, J.; Lee, C.; Doukas, A.G.; Goodson, J.M. Phototargeting oral black-pigmented bacteria. Antimicrob. Agents Chemother. 2005, 49, 1391-1396. [CrossRef]

35. Strakhovskaya, M.G.; Belenikina, N.S.; Ivanova, E.; Ivanova, E.V.; Chemeris, Y.K.; Stranadko, E.F. The photodynamic inactivation of the yeast Candida guilliermondii in the presence of photodithazine. Microbiology 2002, 71, 298. [CrossRef]

36. Wu, J.; Chu, Z.; Ruan, Z.; Wang, X.; Dai, T.; Hu, X. Changes of intracellular porphyrin, reactive oxygen species, and fatty acids profiles during inactivation of methicillin-resistant Staphylococcus aureus by antimicrobial blue light. Front. Physiol. 2018, 28, 1658. [CrossRef]

37. Amin, R.; Bhayana, B.; Hamblin, M.; Dai, T. Antimicrobial blue light inactivation of Pseudomonas aeruginosa by photo-excitation of endogenous porphyrins: In vitro and in vivo studies. Lasers Surg. Med. 2016, 48, 562-568. [CrossRef]

38. Schmid, J.; Hoenes, K.; Vatter, P.; Hessling, M. Antimicrobial effect of visible light-photoinactivation of Legionella rubrilucens by irradiation at 450, 470 and $620 \mathrm{~nm}$. Antibiotics 2019, 8, 187. [CrossRef]

39. Hoenes, K.; Hess, M.; Vatter, P.; Spellerberg, B.; Hessling, M. $405 \mathrm{~nm}$ and $450 \mathrm{~nm}$ Photoinactivation of Saccharomyces cerevisiae. Eur. J. Microbiol. Immunol. 2018, 4, 142-148. [CrossRef]

40. Plavskii, V.Y.; Mikulich, A.V.; Tretyakova, A.I.; Leusenka, I.A.; Plavskaya, L.G.; Kazyuchits, O.A.; Dobysh, I.I.; Krasnenkova, T.P. Porphyrins and flavins as endogenous acceptors of optical radiation of blue spectral region determining photoinactivation of microbial cells. J. Photochem. Photobiol. 2018, 183, 172-183. [CrossRef]

41. Hoenes, K.; Wenzel, U.; Spellerberg, B.; Hessling, M. Photoinactivation sensitivity of Staphylococcus carnosus to visible-light irradiation as a function of wavelength. Photochem. Photobiol. 2020, 96, 156-169. [CrossRef]

42. Nitzan, Y.; Salmon-Divon, M.; Shporen, E.; Malik, Z. ALA induced photodynamic effects on gram positive and negative bacteria. Photochem. Photobiol. Sci. 2004, 3, 430-435. [CrossRef]

43. Barneck, M.D.; Rhodes, N.L.R.; de la Presa, M.; Allen, J.P.; Poursaid, A.E.; Nourian, M.M.; Firpo, M.A.; Langell, J.T. Violet 405-nm light: A novel therapeutic agent against common pathogenic bacteria. J. Surg. Res. 2016, 206, 316-324. [CrossRef]

44. Gulías, Ò.; McKenzie, G.; Bayó, M.; Agut, M.; Nonell, S. Effective photodynamic inactivation of 26 Escherlichia coli strain with differen antibiotic susceptibility profiles: A planktonic and biofilm study. Antibiotics 2020, 9, 98. [CrossRef]

45. Abana, C.; Brannon, J.; Ebbott, R.; Dunigan, T.; Guckes, K.; Fuseini, H.; Powers, J.; Rogers, B.R.; Hadjifrangiskou, M. Characterization of blue light irradiation effects on pathogenic and nonpathogenic Escherichia coli. Microbiologyopen 2017, 6, 4006-40014. [CrossRef] 
46. Ghate, V.; Leong, A.; Kumar, A.; Bang, W.; Zhou, W.; Yuk, H. Enhancing the antibacterial effect of 461 and $521 \mathrm{~nm}$ light emitting diodes on selected foodborne pathogens in trypticase soy broth by acidic and alkaline pH conditions. Food Microbiol. 2015, 48, 49-57. [CrossRef]

47. Hessling, M.; Spellerberg, B.; Hoenes, K. Photoinactivation of bacteria by endogenous photosensitizers and exposure to visible light of different wavelegths-A review on existing data. FEMS Microbiol. Lett. 2017, 362, 1-12. [CrossRef]

48. Kim, S.W.; Kim, J.; Lim, W.B. In vitro bactericidal effects of 625, 525, and $425 \mathrm{~nm}$ wavelength (red, green, and blue) light-emitting diode irradiation. Photomed. Laser Surg. 2013, 31, 554-562. [CrossRef]

49. Ghate, V.S.; Ng, K.S.; Zhou, W.; Yang, H.; Khoo, G.H.; Yoon, W.-B.; Yuk, H.-G. Antibacterial effect of light emitting diodes of visible wavelengths on selected foodborne pathogens at different illumination temperatures. Int. J. Foor Microbiol. 2013, 166, 399-406. [CrossRef]

50. Hyun, J.-E.; Lee, S.-Y. Antibacterial effect and mechanisms of action of 460-470 nm light-emitting diode against Listeria monocytogenes and Pseudomonas fluorescens on the surface of packaged sliced cheese. Food Microbiol. 2020, 86, 103314. [CrossRef]

51. Maclean, M.; MacGregor, S.; Anderson, J.; Woolsey, G. Inactivation of bacterial pathogens following exposure to light from a 405-nanometer light-emitting diode array. Appl. Environ. Microbiol. 2009, 75, 1932-1937. [CrossRef] [PubMed]

52. Kim, M.; Mikš-Krajnik, M.; Kumar, A.; Ghate, V.; Yuk, H. Antibacterial effect and mechanism of high-intensity $405 \pm 5 \mathrm{~nm}$ light emitting diode on Bacillus cereus, Listeria monocytogenes, and Staphylococcus aureus under refrigerated condition. J. Photochem. Photobiol. 2015, 153, 33-39. [CrossRef] [PubMed]

53. Halstead, F.D.; Thwaite, J.E.; Bu, R.; Laws, T.R.; Raguse, M.; Moeller, R.; Webber, M.A.; Oppenheim, B.A. Antibacterial activity of blue light against nosocomial wound pathogens growing planktonically and as mature biofilms. Public Environ. Health Microbiol. 2016, 82, 13. [CrossRef] [PubMed]

54. Wang, Y.; Wang, Y.; Wang, Y.; Murray, C.K.; Hamblin, M.R.; Hooper, D.C.; Dai, T. Antimicrobial blue light inactivation of pathogenic microbes: State of the art. Drug Resist. Updat 2017, 33-35, 1-22. [CrossRef] [PubMed]

55. Brito Aragão, M.G.; Gonçalves de Araújo Costa, C.A.; Lima, R.A.; Azevedo Rodrigues, L.K.; Duarte, S.; Junqueira Zanin, I. Comparative effect of two red lights on Streptococcus mutans biofilms and assessment of temperature variances in human teeth during in vitro photodynamic antimicrobial chemotherapy. Photobiomodul. Photomed. Laser Surg. 2019, 37, 31-37. [CrossRef] [PubMed]

56. Lui, G.Y.; Roser, D.; Corkish, R.; Ashbolt, N.J.; Stuetz, R. Point-of-use water disinfection using ultrviolet and visible light-emitting diodes. Sci. Total Environ. 2016, 553, 626-635. [CrossRef] [PubMed]

57. Yu, S.; Lee, Y.H. Effect of light quality on Bacillus amyloliquefaciens JBC36 and its biocontrol efficacy. Biol. Control 2013, 64, 203-210. [CrossRef]

58. Yu, S.; Ramkumar, G.; Lee, Y. Light quality influences the virulence and physiological responses of Colletotrichum acutatum causing anthracnose in pepper plants. J. Appl. Microbiol. 2013, 115, 509-516. [CrossRef]

59. Otter, J.; Vickery, K.; Walker, J.; deLancey Pulcini, E.; Stoodley, P.; Goldenberg, S.; Salkeld, J.A.; Chewins, J.; Yezli, S.; Edgeworth, J.D. Surface-attached cells, biofilms and biocide susceptibility: Implications for hospital cleaning and disinfection. J. Hosp. Infect. 2015, 89, 16-27. [CrossRef]

60. Nogarol, C.; Acutis, P.; Bianchi, D.; Maurella, C.; Peletto, S.; Gallina, S.; Adriano, D.; Zuccon, F.; Borrello, S.; Caramelli, M.; et al. Molecular characterization of Pseudomonas fluorescens isolates involved in the Italian "blue mozzarella" event. J. Food Prot. 2013, 76, 500-504. [CrossRef]

61. Eladli, M.G.; Alharbi, N.S.; Khaled, J.M.; Kadaikunnan, S.; Alobaidi, A.S.; Alyahya, S.A. Antibiotic-resistant Staphylococcus epidermidis isolated from patients and healthy students comparing with antibiotic-resistant bacteria isolated from pasteurized milk. Saudi J. Biol. Sci. 2019, 26, 1285-1290. [CrossRef] [PubMed]

62. Geeraerd, A.H.; Herremans, C.H.; Van Impe, J.F. Structural model requirements to describe microbial inactivation. Int. J. Food Microbiol. 2000, 59, 185-209. [CrossRef]

63. Murdoch, L.; McKenzie, K.; Maclean, M.; Macgregor, S.; Anderson, J. Lethal effects of high-intensity violet 405-nm light on Saccharomyces cerevisiae, Candida albicans, and on dormant and germinating spores of Aspergillus niger. Fungal Biol. 2013, 117, 519-527. [CrossRef] [PubMed] 
64. Maclean, M.; McKenzie, K.; Anderson, J.G.; Gettinby, G.; MacGregor, S.J. 405 nm light technology for the inactivation of pathogens and its potential role for environmental disinfection and infection control. J. Hosp. Infect. 2014, 88, 1-11. [CrossRef]

65. Meurer, L.; Payne, W.; Guffey, J.S. Visible light as an inhibitor of Campylobacter Jejuni. Int. J. Antimicrob. Agents 2020, 55, 1. [CrossRef]

66. Schmid, J.; Hoenes, K.; Rath, M.; Vatter, P.; Spellerberg, B.; Hessling, M. Photoinactivation of Legionella Rubrilucens by visible light. Eur. J. Microbiol. Immunol. 2017, 7, 146-149. [CrossRef]

67. Tomb, R.M.; White, T.A.; Coia, J.E.; Anderson, J.G.; MacGregor, S.J.; Maclean, M. Review of the comparative susceptibility of microbial species to photoinactivation using $380-480 \mathrm{~nm}$ violet-blue light. Photochem. Photobiol. 2018, 96, 445-458. [CrossRef]

68. Kumar, A.; Ghate, V.; Kim, M.-J.; Zhou, W.; Khoo, G.; Yuk, H.-G. Inactivation and changes in metabolic profile of selected foodborne bacteria by $460 \mathrm{~nm}$ LED illumination. Food Microbiol. 2017, 63, 12-21. [CrossRef]

69. Carmello, J.; Dovigo, L.; Mima, E.; Jorge, J.; de Souza Costa, C.; Bagnato, V.S.; Pavarina, A.C. In vivo evaluation of photodynamic inactivation using Photodithazine ${ }^{\circledR}$ against Candida albicans. Photochem. Photobiol. Sci. 2015, 14, 1319-1328. [CrossRef]

70. Ogonowska, P.; Woźniak, A.; Pierański, M.K.; Nakonieczna, J. Application and characterization of light-emitting diodes for photodynamic inactivation of bacteria. Lighting Res. Technol. 2018, 1-13. [CrossRef]

71. Fernández, R.; Pizarro, R. Lethal effect induced in Pseudomonas aeruginosa exposed to ultraviolet-A radiation. Photochem. Photobiol. 1996, 64, 334-339. [CrossRef] [PubMed]

72. Magro, M.; Baratella, D.; Jakubec, P.; Corraducci, V.; Fasolato, L.; Cardazzo, B.; Novelli, E.; Zoppellaro, G.; Zboril, R.; Vianello, F. $\mathrm{H}_{2} \mathrm{O}_{2}$ tolerance in Pseudomonas fluorescens: Synergy between pyoverdine-iron(III) complex and a blue extracellular product revealed by a nanotechnology-based electrochemical approach. ChemElectroChem 2019, 6, 5186-5190. [CrossRef]

73. Kogan, B.Y. Nonlinear photodynamic therapy. Saturation of a photochemical dose by photosensitizer bleaching. Photochem. Photobiol. Sci. 2003, 6, 673-676. [CrossRef] [PubMed]

74. Rupel, K.; Zupin, L.; Ottaviani, G.; Bertani, I.; Martinelli, V.; Porrelli, D.; Vodret, S.; Vuerich, R.; Passos da Silva., D.; Bussani, R.; et al. Blue laser light inhibits biofilm formation in vitro and in vivo by inducing oxidative stress. Biofilms Microbiomes 2019, 5, 1. [CrossRef] [PubMed]

75. Busch, S.; Donnelly, C. Development of a repair-enrichment broth for resuscitation of heat-injured Listeria monocytogenes and Listeria innocua. Appl. Environ. Microbiol. 1992, 58, 14-20. [CrossRef] 